\title{
Eddies in Numerical Models of the Southern Ocean
}

\author{
V.O. Ivchenko', S. Danilov, and D. Olbers \\ Alfred Wegener Institute for Polar and Marine Research Bremerhaven, Germany
}

\begin{abstract}
Mesoscale eddies play a crucial role in the dynamics of the Antarctic Circumpolar Current (ACC) by facilitating horizontal redistribution and vertical penetration of the momentum, contributing to the meridional heat transport and the budgets of energy and momentum of the ACC. This chapter discusses the dynamics of the ACC based on results of numerical models that permit or resolve the mesoscale eddies. Conclusions are drawn by comparing and contrasting results from different models, including both quasi-geostrophic and primitive equation models.
\end{abstract}

\section{INTRODUCTION}

The Southern Ocean is a unique part of the World Ocean. Its essential and most important feature is the Antarctic Circumpolar Current (ACC), the strongest current of the World Ocean transporting about 130 to $150 \mathrm{~Sv}\left(1 \mathrm{~Sv}\right.$ is $\left.10^{6} \mathrm{~m}^{3} / \mathrm{s}\right)$ of water. It circumnavigates the Antarctic continent, as there are no meridional barriers blocking it completely. The circumpolar character of the ACC imposes constraints on its dynamics, whereby eddies play an outstanding role in meridional and vertical transfers. Plate 1, adopted from Hallberg and Gnanadesikan [2006], illustrates how the complex flow pattern containing numerous small-scale eddies in the ACC emerges as resolution of models increases. We will describe three major ways the eddies influence the ACC dynamics.

The first of them is linked to the fact that the zonally averaged geostrophic meridional velocity is zero in the unconstrained belt of latitudes in the Southern Ocean. Thus, the meridional exchange of water, heat, salt, and other substances between the Southern Ocean, Atlantic, Indian, and Pacific Oceans takes place via the eddy exchanges.

${ }^{1}$ Now at National Oceanography Centre, Southampton, UK.

Ocean Modeling in an Eddying Regime

Geophysical Monograph Series 177

Copyright 2008 by the American Geophysical Union.

10.1029/177GM13
1. Eddies are a major contributor in meridional exchanges in the Southern Ocean.

As a consequence, they provide an important input into the global thermohaline circulation [Rintoul et al., 2001, Bryden and Cunningham, 2003].

The ACC is mainly eastward; however, it deviates from the zonal direction on passing the main topographic features (see Plate 1). A positive zonal component of the mean velocity is observed at all vertical levels from the surface to the bottom. A strong westerly wind above the ACC inputs eastward momentum into the current. The eastward momentum penetrates down to the bottom through the action of interfacial form stress. It occurs in vertically stratified sheared flows with undulating density surfaces in a manner similar to the topographic form stress in flows over topography [Johnson and Bryden, 1989; Marshall et al., 1993; Ivchenko et al., 1996]. Eddies displace the isopycnal surfaces from their mean positions and are therefore indispensable in setting the momentum balance. This is the second major way the eddies influence the ACC dynamics:

2. Eddies enable the downward penetration of momentum in the ACC via the interfacial form stress. It is linked to the meridional eddy mass flux.

The ACC is a multijet system with variable positions of the jets in both space and time (see Plate 1). Moreover, the number of jets varies along the path of the ACC. Eddies mix the potential vorticity (PV) in various subdomains along the path of the ACC and create and maintain main fronts and their local branches in the Southern Ocean. The energy budget of the ACC is very different from the energy budget of the main 
178 EDDIES IN NUMERICAL MODELS OF THE SOUTHERN OCEAN

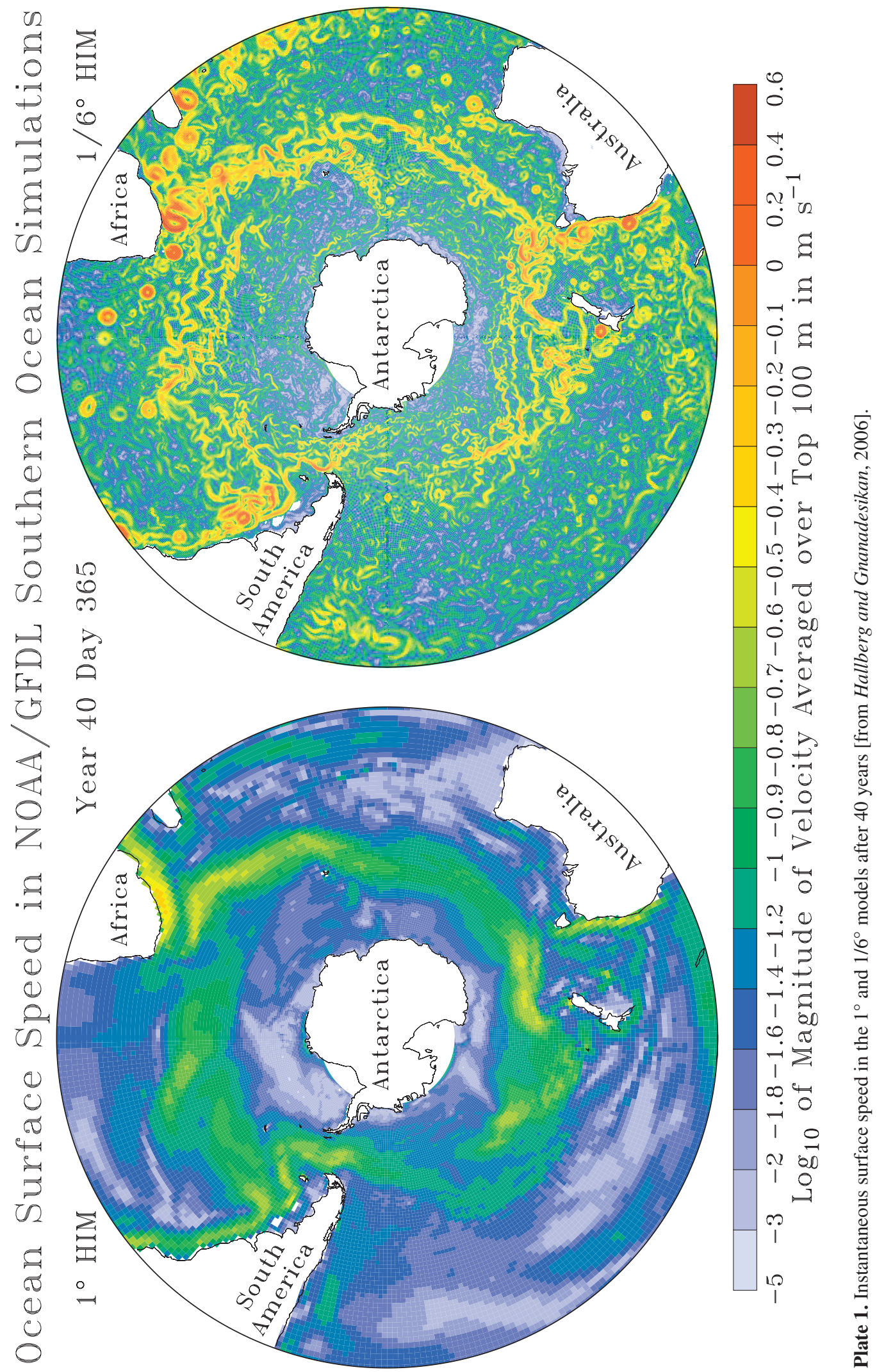


gyres in ocean basins. The eddy contribution is a major constituent in the Southern Ocean. Correspondingly, the third major way the eddies contribute in the ACC dynamics is:

3. Eddies redistribute momentum, potential vorticity, and energy and also create/maintain the fronts.

These three issues are described in more detail below. Many results are based on the analysis of the output of eddy-resolving numerical models. The first baroclinic Rossby radius in the Southern Ocean is varying and decreases poleward to values smaller than $10 \mathrm{~km}$ because of a weak stratification. This demands high horizontal resolution from a numerical model. For this reason, the first eddyresolving model of the ACC was a quasi-geostrophic (QG) model set up for an idealized channel with only two layers and reduced zonal length [McWilliams et al., 1978]. This and the other studies based on QG equations illuminated many aspects of the ACC dynamics such as the impact of bottom topography, dependence of the flow on horizontal scales of the channel, and so on [Treguier and McWilliams, 1990; Wolff et al., 1991]. In spite of their apparent limitations, they provided a valuable insight into interaction between eddies and mean quasi-zonal flow in "the numerical ACC."

More recent experiments were based on primitive equation models [Semtner and Chervin model, Fine-Resolution Antarctic model (FRAM), Ocean Circulation and Climate Advanced Model (OCCAM), Parallel Ocean Program (POP), Modeling Eddies in the Southern Ocean] working on eddy-permitting or resolving meshes [Semtner and Chervin, 1988, 1992; The FRAM Group, 1991; Webb et al., 1998; Maltrud et al., 1998; Maltrud and McClean, 2005; Hallberg and Gnanadesikan, 2006]. All of the models have a good vertical resolution and realistic bottom topography. Forced with realistic wind stresses and surface heat and freshwater fluxes, these models are capable of reproducing the dynamics of the ACC and other processes in the Southern Ocean with a growing degree of realism.

There are many similarities as well as differences in the results of simulations with these models. Comparing and contrasting them allows better understanding of the dynamics of the Southern Ocean and the role played by eddies there.

In this chapter, we are discussing only those aspects of the Southern Ocean dynamics that are directly linked to the eddy activity. Some more indirect effects of eddies hidden in water mass formation and spreading, interaction with the atmosphere, as well as parameterization of the eddy fluxes are not discussed here.

\section{QG EDDY-RESOLVING MODELS IN A ZONAL CHANNEL}

The progress in early studies of eddy dynamics in zonal flows and eddy interactions with mean flows and topography relies on using QG models, and our current understanding of the ACC dynamics owes much to such studies [McWilliams et al., 1978; McWilliams and Chow, 1981; Wolff and Olbers, 1989; Treguier and McWilliams, 1990; Wolff et al., 1991]. A number of experiments were conducted with flows in rectangular channels of different zonal extent with or without topographic obstacles and driven by different wind stresses. The basic questions answered with QG models concern momentum balance, vertical penetration of momentum, and the convergence of zonal momentum in eastward jets. The total zonal transport as well as the pattern of the mean flow and eddy activity prove to be strongly dependent on the presence and details of the bottom topography.

\subsection{Flat Bottom Zonal Channel}

In a flat-bottom zonal channel, the momentum imparted by wind to the upper layer is transferred down by interfacial form stress. It can be balanced only by bottom friction if the lateral friction at the side walls is small. This leads to unrealistically high values of the total transport, which is about one order of magnitude higher than the observed ACC transport.

In the absence of forcing and dissipation, the time variation of the depth-integrated zonally averaged zonal momentum is determined by the depth-integrated meridional eddy flux of quasi-geostrophic potential vorticity (QPV) $[\mathrm{Ped}$ losky, 1979]. In a two-layer model, this is expressed as

$$
\frac{\partial}{\partial t}\left(H_{1} \bar{u}_{1}^{x}+H_{2} \bar{u}_{2}^{x}\right)=H_{1}{\overline{v_{1}^{\prime} q_{1}^{\prime}}}^{x}+H_{2}{\overline{v_{2}^{\prime} q_{2}^{\prime}}}^{x}
$$

where $u_{i}, v_{i}$ are the zonal and meridional components of the horizontal velocity, respectively; subscripts 1 and 2 mark the upper and lower layers whose mean thicknesses $H_{i}$ are constant. $q_{i}$ is the QPV,

$$
q_{i}=\nabla^{2} \psi_{i}+f+(-1)^{i} \frac{f_{0}^{2}}{g^{\prime} H_{i}}\left(\psi_{1}-\psi_{2}\right),
$$

where $\psi$, represents the horizontal geostrophic velocity streamfunctions, $u_{i}=-\partial \psi_{i} / \partial y, v_{i}=\partial \psi_{i} / \partial x ; f$ is the Coriolis parameter, and $f_{0}$ its reference value; $g^{\prime}=g\left(\rho_{2}-\rho_{1}\right) / \rho_{0}$ is the reduced gravity; $\rho_{i}$ is the constant density in layer $i$, and $\rho_{0}$ is the reference density; $g$ is the acceleration due to gravity. The overbar with $x$ mark and prime denote the zonal average and eddy component (the deviation from the zonal mean), respectively. It is straightforward to show that the integral over the meridional extent of the channel of the 
depth-integrated meridional eddy QPV flux is zero for a flatbottom channel [Bretherton, 1966]:

$$
\int_{0}^{L}\left(H_{1}{\overline{v_{1}^{\prime} q_{1}^{\prime}}}^{x}+H_{2}{\overline{v_{2}^{\prime} q_{2}^{\prime}}}^{x}\right) d y=0
$$

where $y=0$ is the southern boundary, and $L$ is the width of the channel.

Expressions (1) and (3) imply that eddies do not change the total zonal momentum:

$$
\frac{d}{d t} \int_{0}^{L}\left(H_{1} \bar{u}_{1}^{x}+H_{2} \bar{u}_{2}^{x}\right) d y=0 .
$$

In experiments with a flat bottom, there is no substantial variability in the ACC transport and no standing (stationary in time) eddies because of temporal and zonal invariance. The transient eddies can contain patterns propagating zonally, but are independent (on average) of the zonal coordinate. A strong zonal mean jet forms in each layer with a maximum in the center when the sin-type distribution of zonal wind stress is applied (Figure 1). The upper jet is stronger because the eddy-induced lateral Reynolds stress transfers the eastward momentum to its center, making it narrower and more intense [Held, 1975].

A necessary condition for the baroclinic instability in a two-layer QG model is the difference in signs of the mean meridional QPV gradients [Pedlosky, 1979], given by:

$$
\frac{\partial{\overline{q_{i}}}^{x}}{\partial y}=-\frac{\partial^{2}{\overline{u_{i}}}^{x}}{\partial y^{2}}+\beta-(-1)^{i} \frac{f_{0}^{2}}{g^{\prime} H_{i}}\left({\overline{u_{1}}}^{x}-{\overline{u_{2}}}^{x}\right),
$$

where $\beta=\partial f / \partial y$. The first term on the right-hand side (rhs) of (5) is the meridional gradient of the relative vorticity that is much smaller than the other two terms [Marshall, 1981]. Thus, the sign of the meridional gradient of the QPV in the upper layer is positive everywhere because $\left(\bar{u}_{1}^{x}-\bar{u}_{2}^{x}\right)>0$. In the lower layer, $\partial \bar{q}_{2}^{x} / \partial y$ can change its sign from negative in the center of the jet to positive at the periphery. The negative value of $\partial \bar{q}_{2}^{x} / \partial y$ corresponds to a baroclinic instability and is limited to the central part of the channel where

$$
\left({\overline{u_{1}}}^{x}-{\overline{u_{2}}}^{x}\right)>\frac{\beta g^{\prime} H_{2}}{f_{0}^{2}} .
$$

Note that the criterion of instability for the zonal westward flow is

$$
\left({\overline{u_{1}}}^{x}-{\overline{u_{2}}}^{x}\right)<-\frac{\beta g^{\prime} H_{1}}{f_{0}^{2}} .
$$
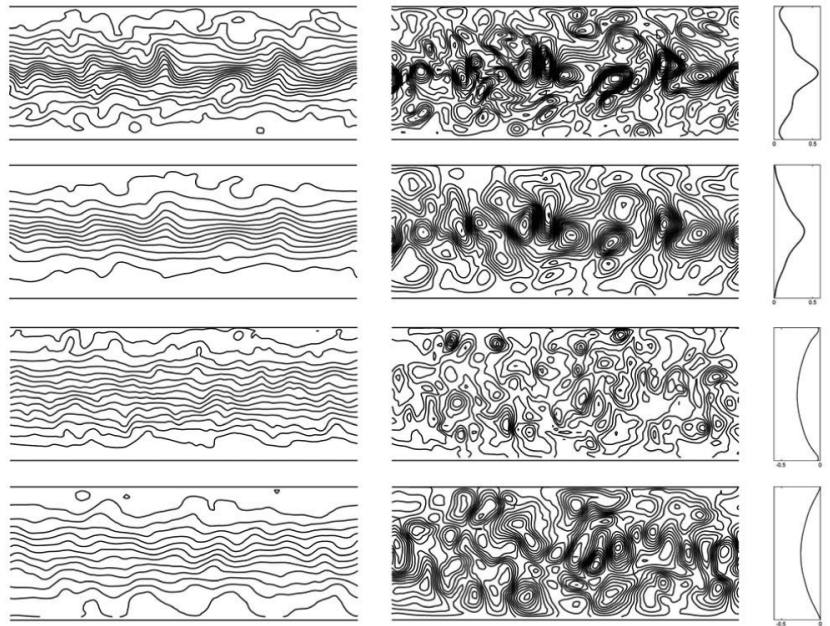

Figure 1. (a) Circulation for eastward wind stress in a flat-bottom QG two-layer model; (b) circulation for westward wind stress [from Olbers, 2005]. In each set the contours of the instantaneous (left upper panel: first layer; left lower panel: second layer) and eddy streamfunction (middle upper panel: first layer; middle lower panel: second layer) are displayed as well as the meridional velocity profiles of the zonal-time mean (right panels). Contour intervals are $2 \times 10^{4} \mathrm{~m}^{2} \mathrm{~s}^{-1}$ for the instantaneous flow (both layers) and $5 \times$ $10^{3} \mathrm{~m}^{2} \mathrm{~s}^{-1}$ for the eddy flow in the first layer and $2.5 \times 10^{3} \mathrm{~m}^{2} \mathrm{~s}^{-1}$ for the second layer. Velocity is expressed in $\mathrm{m} \mathrm{s}^{-1}$.

The upper layer represents the main thermocline with a depth scale of 1,000 m, while the lower one corresponds to the deep ocean with a scale of about $4,000 \mathrm{~m}$, that is, $H_{1}<$ $H_{2}$. The difference in thicknesses implies that the westward flow is more unstable than the eastward flow [Kamenkovich et al., 1986]. Although only the eastward flow simulates the ACC, it is illuminating to compare it with the westward flow, following Olbers [2005], to highlight the role of baroclinic instability in shaping these flows.

The westward flow becomes unstable at smaller amplitudes of the vertical shear, which explains smaller values of available potential energy (APE) of the mean flow and less vigorous eddies. The patterns of the eastward and westward currents differ significantly (Figure 1). The westward current is not a narrow jet, as the eastward flow, but is wide and smooth, with a typical meridional scale close to the meridional scale of the zonal wind stress. In the eastward flow, eddies transport the eastward momentum by Reynolds stress $\overline{u_{1}^{\prime} v_{1}^{\prime}}$ to the jet center both from north and from south (Figure 1a). For the westward flow, they transfer westward momentum to the jet center, but this effect is not large and does not result in a jet concentration [Ivchenko et al., 1997a] (Figure 1b). 
The most striking difference is seen in the steady-state APE of the mean flow (671 and $145 \mathrm{~m}^{3} \mathrm{~s}^{-2}$ for the eastward and westward flows, respectively). The eddies in the upper layer are more energetic in the eastward flow with the eddy kinetic energy (EKE) $21 \mathrm{~m}^{3} \mathrm{~s}^{-2}$ compared to $5 \mathrm{~m}^{3} \mathrm{~s}^{-2}$ in the westward flow. The eddy APE in the eastward flow exceeds that of the westward flow almost threefold $\left(28\right.$ and $10 \mathrm{~m}^{3}$ $\mathrm{s}^{-2}$ ). The quantities reported here are water column or layerintegrated values.

\subsection{Zonal Channel With Bottom Topography}

A major modification introduced by the bottom topography is that the bottom form stress balances the forcing. This mechanism is inviscid and proves to be very effective in drastically reducing the total zonal transport compared to the flat bottom case. Indeed, the Bretherton theorem (3) in this case is rewritten as [Ivchenko, 1987; Vallis, 2006]:

$$
\int_{0}^{L}\left(H_{1}{\overline{v_{1}^{\prime} q_{1}^{\prime}}}^{x}+H_{2}{\overline{v_{2}^{\prime} q_{2}^{\prime}}}^{x}\right) d y=f_{0} \int_{0}^{L}{\overline{v_{2} b}}^{x} d y
$$

Here, $b$ is the bottom relief measured relative to the unperturbed constant depth of the lower layer $\mathrm{H}_{2}$. The term under the integral on the rhs of (8) is the topographic form stress, as

$$
f_{0}{\overline{v_{2} b}}^{x}=-\bar{p}_{2} \frac{\partial b}{\partial x}
$$

where $p$ is the pressure.

Experiments show that even a small zonal variation in $b$ substantially reduces the zonal transport. For example, random depth variability with root mean square (rms) height of about $200 \mathrm{~m}$ generates the bottom form stress sufficient to replace the bottom friction in the momentum budget [Treguier and McWilliams, 1990]. Isolated features with the same rms height produce even stronger topographic form stress.

In a zonal channel configuration with topography, one separates the eddy field into standing and transient components, with the former representing the time-averaged departure from the zonal mean. Depending on the height and shape of the bottom topography, the standing eddies can play an extraordinary role in the energy budgets and downward penetration of the zonal momentum. The zonal transport in the lower layer can be even negative for some realizations of bottom topography [Treguier and McWilliams, 1990; Wolff et al., 1991].

Experiments with the QG models have shed light on the most important processes in the ACC dynamics involving eddies: The vertical penetration and horizontal redistribution of the zonal momentum occur through the interfacial form stress and Reynolds stress created by transient and standing eddies. Topographic form stress mainly balances the input of momentum by wind stress. However, the QG scaling imposes severe limitations that can distort the physics of large-scale dynamics: For example, an absence of outcropping isopycnals does not allow producing correct meridional circulation and meridional tracer and water mass propagation. Such limitations can be lifted using primitive equation models.

\section{PRIMITIVE EQUATION MODELS}

Numerical models of the Southern Ocean can be either regional, that is, considering only the Southern Ocean, or global. The open boundary of regional models can potentially influence the circulation within the computational domain. An obvious advantage of regional modeling is computational efficiency, as a large part of the World Ocean is excluded.

Among the primitive equation models discussed here, the FRAM [The FRAM Group, 1991] is a regional one. The other models are global. They include the OCCAM [Webb et al., 1998] and the POP model [Maltrud et al., 1998].

The horizontal resolution of the FRAM model is $0.5^{\circ}$ by $0.25^{\circ}$ in zonal and meridional directions, respectively, and it has 32 vertical levels. The vertical grid spacing varies from $20 \mathrm{~m}$ at the surface to $230 \mathrm{~m}$ close to the bottom. The model covers the region from $78^{\circ} \mathrm{S}$ to $23^{\circ} \mathrm{S}$. Its bottom topography is smoothed at $1^{\circ}$. The model has been run for 16 years and the analysis uses data from the last 6 years.

The OCCAM exists at three different horizontal resolutions of $1^{\circ}, 1 / 4^{\circ}$, and $1 / 12^{\circ}$. The resolution of $1^{\circ}$ is too coarse to resolve eddies, $1 / 4^{\circ}$ is "eddy-permitting," and $1 / 12^{\circ}$ is eddy-resolving. The finest version is very demanding with respect to computer resources and was run only over a limited period. The $1 / 4^{\circ}$ model has 36 vertical levels. It was integrated over 12 years, and the analysis was made for the last 4 years.

The results of the POP model presented here were obtained on the Mercator grid with the horizontal size changing between $31.25 \mathrm{~km}$ at the equator and $6.8 \mathrm{~km}$ at $77^{\circ} \mathrm{N}$ or $\mathrm{S}$ and having 20 vertical layers [Smith et al., 1992; Dukowicz et al., 1993; Dukowicz and Smith, 1994; Maltrud et al., 1998]. Such a fine horizontal resolution in high latitudes makes this version a good choice for studies of the Southern Ocean.

Several other numerical studies have appeared recently at horizontal resolution which is superior to that used in the models mentioned above [see Maltrud and McClean, 2005; Hallberg and Gnanadesikan, 2006]. Yet, our choice here is 
limited to models and simulations for which thorough analyses of eddy contributions were made.

Discussing the dynamics of the Southern Ocean, we have to specify the domain of analysis. The southern boundary is obvious - it is the Antarctic coast. The northern boundary varies in different studies. Even more care is required in selecting the ACC domain. Both "boundaries" of the ACC are open and vary in time and space. In the Drake Passage, there is a belt of latitudes without continental barriers (we will call it the ACC Belt, or ACCB). The problem is that the ACC is not confined to this belt in many places along its path (see Figure 2). This results in much smaller zonal mean transport of the ACC than the mean transport through the Drake Passage. Also a significant part of kinetic energy of the ACC, both of mean flow and eddies, lies outside the ACCB. In FRAM, $80 \%$ of the EKE is generated on the northern flank of the current outside the ACCB [Ivchenko et al., 1996].

Another possibility is to integrate along the time mean path of the ACC transport (we will use the abbreviation ACCP). This approach allows areas such as the northern flank of the ACC to be included in the analysis. The transport in the ACCP coincides with the total transport. A disadvantage of the ACCP approach is that various models produce different positions of streamlines depending on their topography, surface forcing, and resolution. Hence, the integration along streamlines deals with geographically different locations. Both approaches are valuable, as they give complementary views on the ACC dynamics and should be studied together.

\section{MOMENTUM PENETRATION AND THE DEPTH- INTEGRATED BALANCE IN THE ACC}

\subsection{Zonal Balances}

The zonal momentum is imparted to the ACC by strong eastward winds (typical stress values are about $0.1 \mathrm{~N} / \mathrm{m}^{2}$ ). Interfacial form stresses in the ocean are large: Their magnitude is comparable to that of the wind stress [Ferreira et al., 2005]. The sink of the momentum of the depth-averaged flow could be either viscous (by lateral viscosity or bottom friction) or inviscid (by bottom form stress). Estimates show that the balance between the wind stress and any frictional term can be achieved only upon assuming unrealistically high friction. In addition, the eddy meridional flux of the zonal momentum should be too high to provide a balance with the wind stress [Bryden and Heath, 1985]. Munk and Palmen [1951] were the first to formulate the now generally accepted

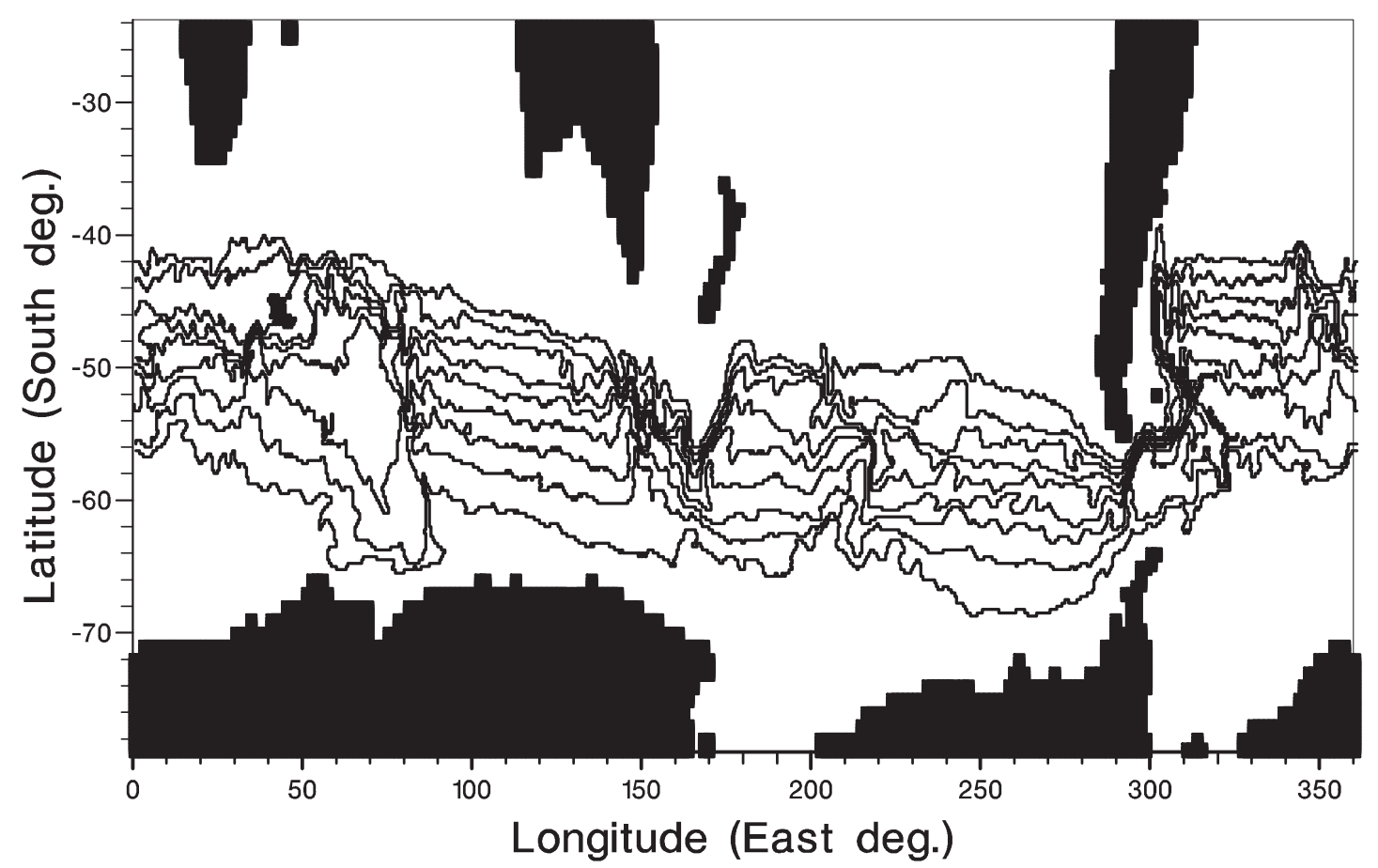

Figure 2. The time-averaged barotropic streamfunction of the ACC from the FRAM. The streamlines range from 10 to $170 \mathrm{~Sv}$ with an interval of $10 \mathrm{~Sv}$. The 10-Sv contour is farthest north [from Ivchenko et al., 1996]. 
view that the topographic form stress $I_{\text {topogr }}={\overline{p^{b}}}_{x}^{x t}$ balances the wind stress $\bar{\tau}_{0}^{x t}$ :

$$
I_{\text {topogr }}+{\overline{\tau_{0}}}^{x t}=0
$$

where the overbar with an $x t$ mark denotes the zonal and time average; $p^{b}$ and $b$ are the bottom pressure and bottom relief, respectively.

Figure 3 shows the balance taken over the final 2 years of the FRAM run [Stevens and Ivchenko, 1997]. Compared to the wind stress or topographic form stress, the poleward momentum-flux divergence and the remaining terms are small. A similar balance is found in the OCCAM and the POP model. However, there are some differences between the balances of these three models [Grezio et al., 2005]. The values of the leading terms in the OCCAM are almost twice as large as in the FRAM and significantly higher then those in the POP model. This is not surprising and is mostly linked to the wind stress. The eastward component of the wind stress used in the OCCAM is based on the European Center for Medium Range Forcasting climatology, which exceeds that of the Hellerman-Rosenstein climatology used by FRAM by a factor of about 2 . The zonal average of the wind stress used in the POP model is lower than that in the OCCAM by about $21 \%$ [Grezio et al., 2005]. This difference is caused by the difference in methods of calculating the stress and in data sets.

The vertical penetration of momentum occurs through the action of interfacial form stress. The higher (lower) pressure is found on the upstream (downstream) side of a rise (fall) in the height of a density surface. The expression for the interfacial form stress can be derived by integrating momentum equations along a constant density surface [Killworth and Nanneh, 1994]. It is also possible to derive a proxy term for the interfacial form stress for a $z$-coordinate model [Johnson and Bryden, 1989; Marshall et al., 1993;

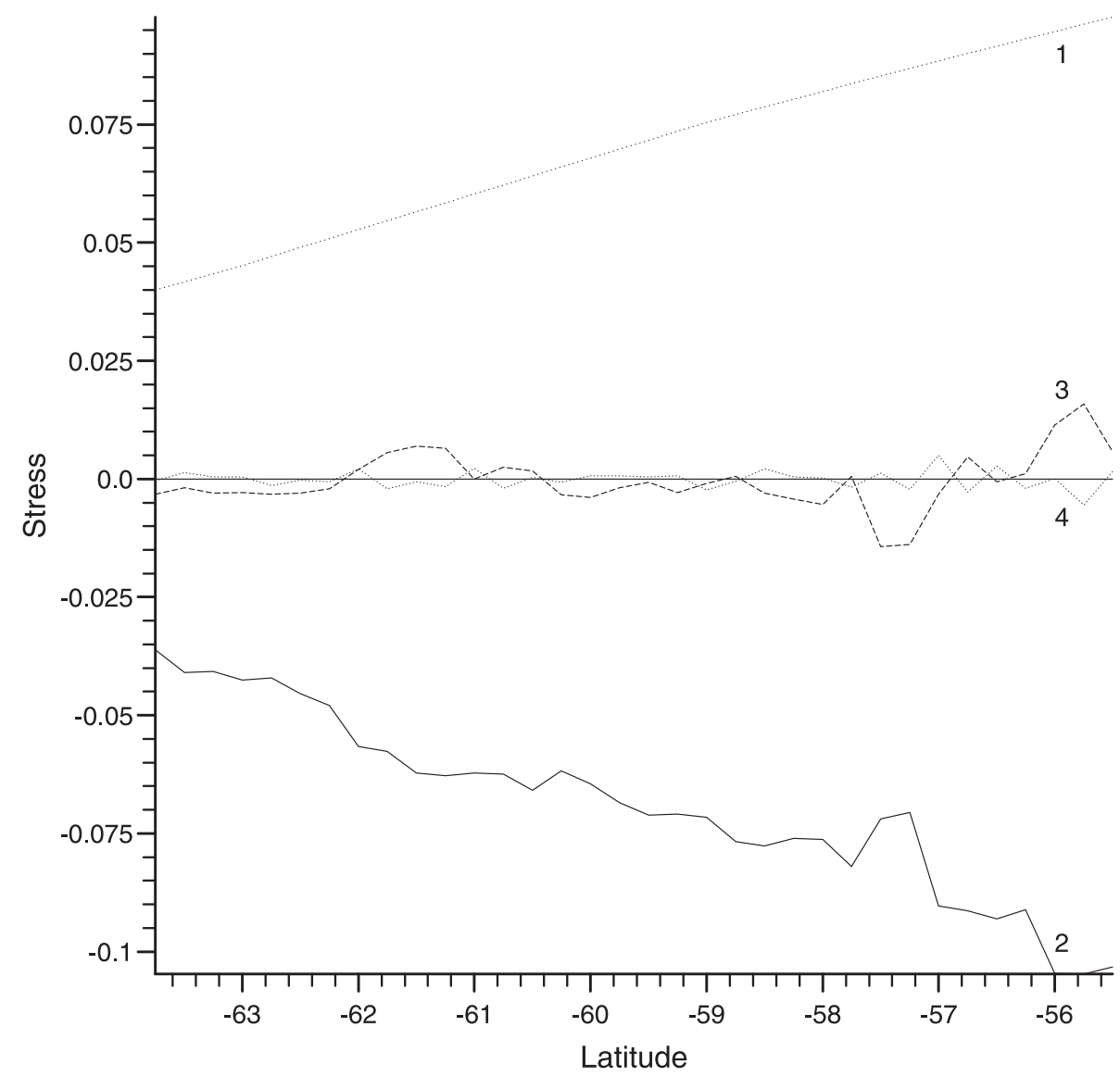

Figure 3. The depth-integrated, time, and zonally averaged momentum balance. Lines 1, 2, 3, and 4 represent the zonal wind stress, bottom form stress, poleward momentum-flux divergence, and remaining (small) terms, respectively [from Stevens and Ivchenko, 1997]. 
Stevens and Ivchenko, 1997]. The interfacial form stress I can be written as

$$
I={\overline{p^{\prime}}}_{\frac{\partial \zeta^{\prime}}{\partial x}}
$$

where $p$ and $\zeta$ are the pressure and the vertical displacement of a constant density surface, respectively; the prime denotes departure from the zonal and time average. The expression for the interfacial form stress $I$ can be rewritten as

$$
I={\overline{p^{\prime}}}_{\frac{\partial \zeta^{\prime}}{\partial x}}^{x t}=\rho_{0} f \frac{{\overline{\rho^{\prime} v^{\prime}}}^{x t}}{{\overline{\rho_{z}}}^{x t}},
$$

on assumption of proportionality between the vertical displacement of density surface and the corresponding density variation [Johnson and Bryden, 1989]. Note that the contributions from both the transient eddies and "standing eddies" (deviation from the zonal mean) define the displacements and thus the interfacial form stress.

The Eliassen-Palm theory provides a powerful method for diagnosing the influence of eddies on zonal mean flows [Eliassen and Palm, 1961; Andrews and McIntyre, 1976; Edmon et al., 1980]. According to this theory, the total eddy influence on the zonal mean flow can be combined in the zonal momentum equation:

$$
-f v_{r}-F=\nabla \cdot \mathbf{E}
$$

where

$$
\mathbf{E}=\left(E^{y}, E^{z}\right)=\left(-{\overline{u^{\prime} v^{\prime}}}^{x t}, f \frac{{\overline{v^{\prime} \rho^{\prime}}}^{x t}}{{\overline{\rho_{z}}}^{x t}}\right)
$$

Here, $v_{r}$ is the meridional component of the "residual velocity" explained below. The term $F$, including friction and

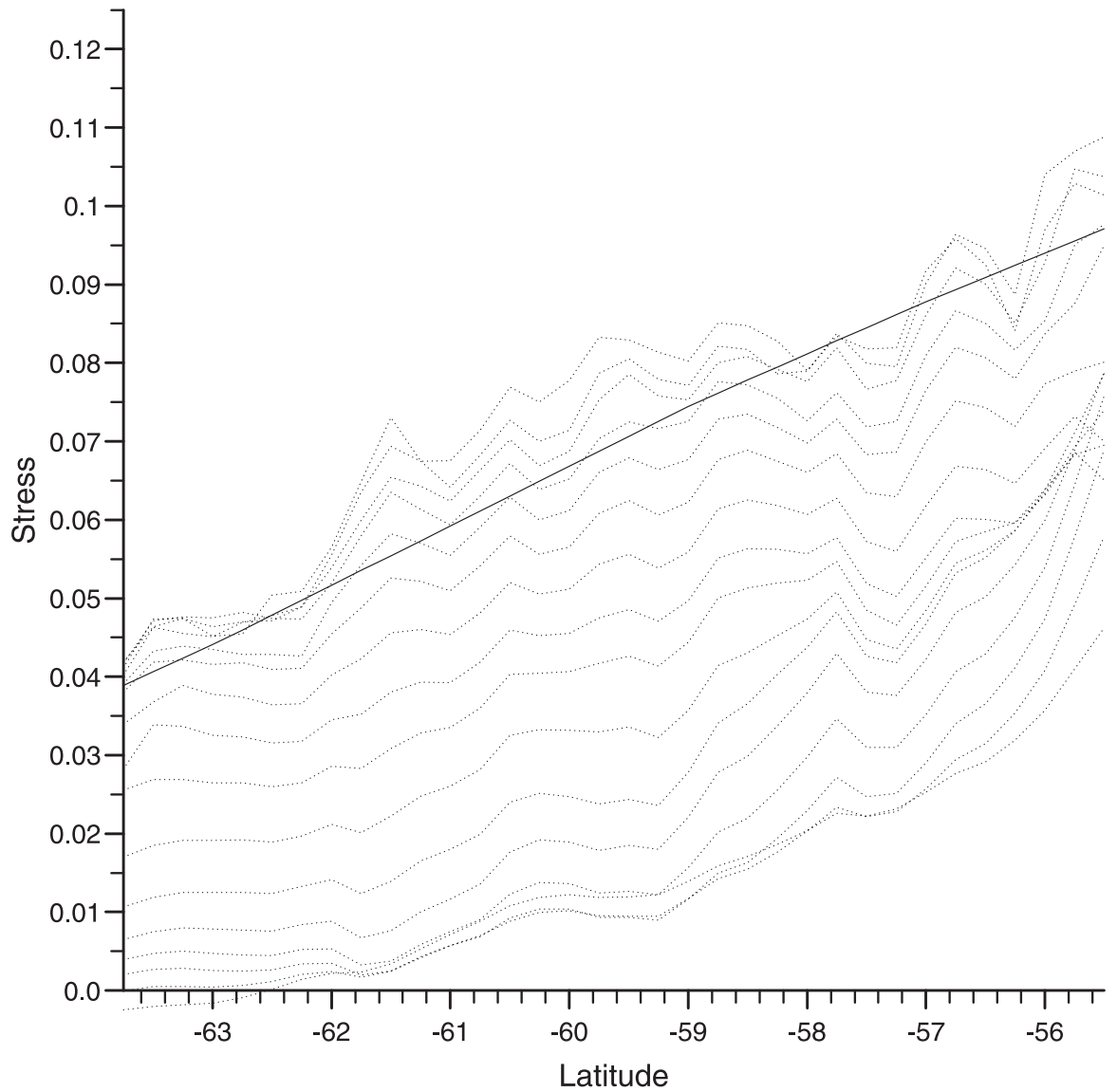

Figure 4. A comparison between the scaled eddy density flux (interfacial form stress $E_{z}$ ) at levels $2-17$ of the FRAM (dotted lines) and the zonal and time averaged eastward wind stress (solid line). The magnitude of the form stress increases with depth. Between levels 13 and $17(1,000-2,000 \mathrm{~m})$, the form stress approximately equals the wind stress. The units are $\mathrm{N} / \mathrm{m}^{2}$ [from Stevens and Ivchenko, 1997]. 
advection, is small below the wind-driven surface layer and above the topography. The horizontal component $E^{y}$ of the Eliassen-Palm vector is the Reynolds stress, while its vertical component $E^{z}$ is the proxy for the interfacial form stress. A complete set of equations can be written for $\bar{u}^{x t}$ and $\rho^{-x t}$ and the components of the "residual meridional circulation" $v_{r}$, $w_{r}$ where

$$
\begin{aligned}
& v_{r}=\bar{v}^{x t}-\frac{\partial}{\partial z}\left(\frac{{\overline{v^{\prime} \rho^{\prime}}}^{x t}}{{\overline{\rho_{z}}}^{x t}}\right), \\
& w_{r}=\bar{w}^{x t}+\frac{\partial}{\partial y}\left(\frac{{\overline{v^{\prime} \rho^{\prime}}}^{x t}}{{\overline{\rho_{z}}}^{x t}}\right) .
\end{aligned}
$$

As the Eulerian velocity field $\left(\bar{v}^{x t}, \bar{w}^{x t}\right)$ can be represented in terms of streamfunction $\psi_{E u}$, a residual streamfunction can also be introduced, according to (15) and (16). The sec- ond terms in the right-hand side of (15) and (16) represent the eddy-induced components of the residual velocity. The eddies drive the zonal flow by Reynolds stress and interfacial form stress in form of the divergence of the Eliassen-Palm flux. The vertical part dominates in the divergence of the Eliassen-Palm vector [Killworth and Nanneh, 1994]. The residual meridional velocity deviates from the Eulerian meridional velocity by this term, which generally is quite substantial, resulting from a strong dependence of the interfacial form stress on depth (Figure 4) [Ivchenko et al., 1996; Stevens and Ivchenko, 1997]. The negative divergence of the Eliassen-Palm vector is related to the poleward meridional residual velocity in the Southern Hemisphere and thus supports the ACC. If there are no diabatic sources, the residual circulation is zero [Ivchenko et al., 1996; Gallego et al., 2004; Olbers, 2005].

The eddy-induced velocity and components of the EliassenPalm vector $E^{y}$ and $E^{z}$ can be separated into transient and

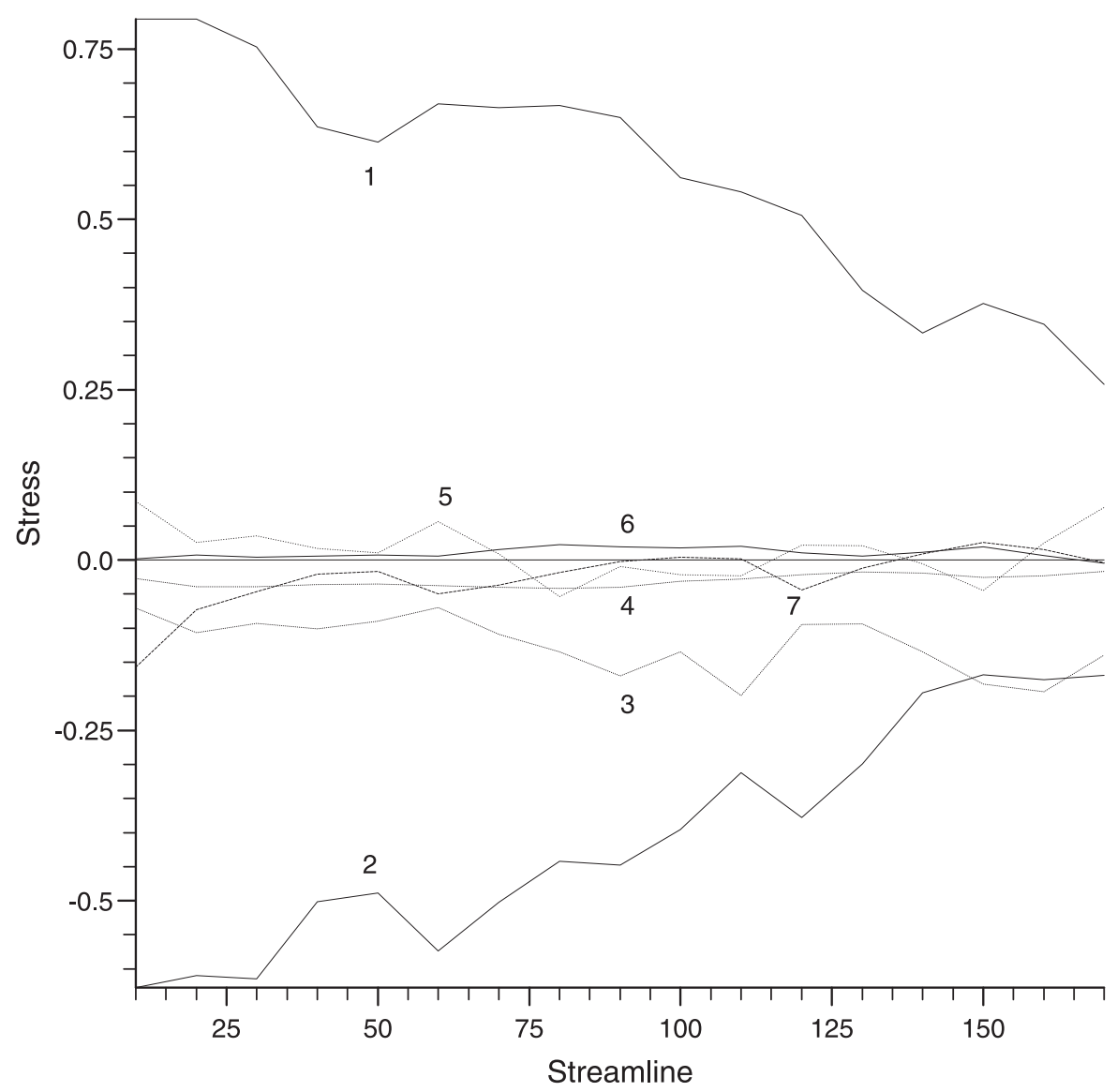

Figure 5. The depth-integrated, time-, and along-streamline-averaged momentum balance in the FRAM. Lines 1, 2, 3, 4, 5, 6, and 7 represent the along-streamline wind stress, bottom form stress, horizontal mixing, bottom friction, horizontal advection of alongstream momentum, vertical advection of along-stream momentum, and remaining (small) terms, respectively. The units are Sv for streamline and dyn $/ \mathrm{cm}^{2}$ for stress [from Ivchenko et al., 1996]. 
standing eddy components. They are of comparable magnitude in models of the Southern Ocean. For the ACCB in the FRAM, the magnitude of the standing eddy component exceeds that of the transient eddy component (see Figures 10 and 12 of Stevens and Ivchenko [1997]). This can be explained by strong meridional deflection of the ACC path from the zonal direction in many places due to bottom topography. The transient eddy activity in the FRAM is smaller than observed values [Ivchenko et al., 1996, 1997b] because of insufficient horizontal resolution. Refining horizontal resolution leads to increasing the relative importance of the transients in the ACCB.

\subsection{Dynamical Balances Along Streamlines}

A way of removing the standing component from the analysis is to consider the ACC dynamics along streamlines [Marshall et al., 1993; Ivchenko et al., 1996; Gille, 1997]. Some of the results obtained for the ACCB are retained for the ACCP. However, there also are substantial differences between balances in the ACCB and ACCP. The wind stress and topographic form stress are still the largest terms in the quasi-zonal (along streamlines) momentum balance in the ACCP computed from the FRAM output. However, other terms are also significant, in particular the horizontal momentum mixing and bottom friction [Ivchenko et al., 1996]. The lateral friction compared to the topographic form stress is not less than $15 \%$, usually $30-50 \%$, and even larger than the form stress at the southern flank of the ACC (Figure 5).

That this is so is not surprising in hindsight. The main jets of the ACC are located north of the ACCB, so the zonal momentum balance is taken over a large region of sluggish water missing much of the dynamics of the ACC. The alongstream momentum balance follows the main jets, and thus, the horizontal mixing and bottom friction terms are larger, while the magnitude of the wind stress remains the same order of magnitude.

Not surprisingly, the expressions for the vertical penetration of the quasi-zonal momentum are similar to the zonal averaging case. There is very strong eddy-induced quasimeridional circulation, which results in a strong deviation of an interfacial form stress from the corresponding mean value of the wind stress [Ivchenko et al., 1996]. The EKE substantially varies along streamlines [Gille, 1997] with highest values associated with the major topographic obstacles.

\section{MERIDIONAL CIRCULATION IN THE SOUTHERN OCEAN}

Analysis of meridional circulation addresses the spreading of water masses within the particular ocean domain and the exchange with the adjacent ocean basins. It is mostly studied in a time-zonal or time-streamline mean and presented by a (meridional) streamfunction describing water exchange in the meridional-vertical plane. Obviously, details of pathways of individual water parcels are lost as well as details of water mass formation. Naturally, the dynamics governing the meridional overturning are identical to the dynamics of the zonal momentum discussed above.

Different types of "zonal" averaging emphasize different properties of the three-dimensional fluid motion. The simplest view on the overturning is obtained from the Eulerian streamfunction $\psi_{E u}(y, z)$ based on the time and zonal mean on $z$ levels. It is governed by the integrated balance of zonal momentum [Olbers and Ivchenko, 2001],

$$
\begin{aligned}
-\rho_{0} f \psi_{E u}(y, z)= & \bar{\tau}_{0}{ }^{x t}(y)-{\overline{\tau^{w}}}^{x t}(y, z) \\
& +I_{\text {topogr }}(y, z)-R(y, z),
\end{aligned}
$$

where ${\overline{\tau^{w}}}^{x t}$ is the time-zonal mean of the zonal stress in the water column and $R$ the integrated Reynolds stress divergence (induced by standing and transient eddies). Consequently, with a small $\bar{\tau}^{w}$ below the mixed layer and small Reynolds stresses, $\psi_{E u}$ generally is dominated by the northward Ekman transport in the surface layers (associated with ${\overline{\tau_{0}}}^{x t}$ ) and a deep geostrophic return flow (associated with $\left.I_{\text {topogr }}\right)$ in the valleys between the highest topography along the particular latitude (see Figure 6a).

This overturning cell is named after Deacon. It does not reflect the more or less adiabatic motion in the ocean interior with transport of active and passive tracers predominantly along isopycnals. This property has more impact on the streamfunction if the zonal average is performed on isopycnals, and indeed the isopycnal streamfunction reveals an essentially different pattern of circulation [see Döös and Webb, 1994; Lee and Coward, 2003; Schouten and Matano, 2006; Hallberg and Gnanadesikan, 2006].

The Eulerian view of the overturning can be extended to a closer correspondence with the isopycnal framework using the transformed Eulerian mean (TEM) approach [Andrews and McIntyre, 1976]. It acknowledges that the transport (advection) of zonally averaged tracers is performed not only by the time-zonal mean flow with streamfunction $\psi_{E u}$ but also by eddies. The eddy contribution can easily be inferred by projecting the mean eddy density flux on directions normal and tangent to the mean isopycnals. The total meridional transport of tracer consists then of three terms: Eulerian overturning, standing, and transient eddy-induced components (see section 4.1). The standing eddy-induced term is easily illustrated by an example of gyre circulation carrying warm (cold) water poleward in the western boundary current and returning cold (warm) water on the eastern part of the gyre. 

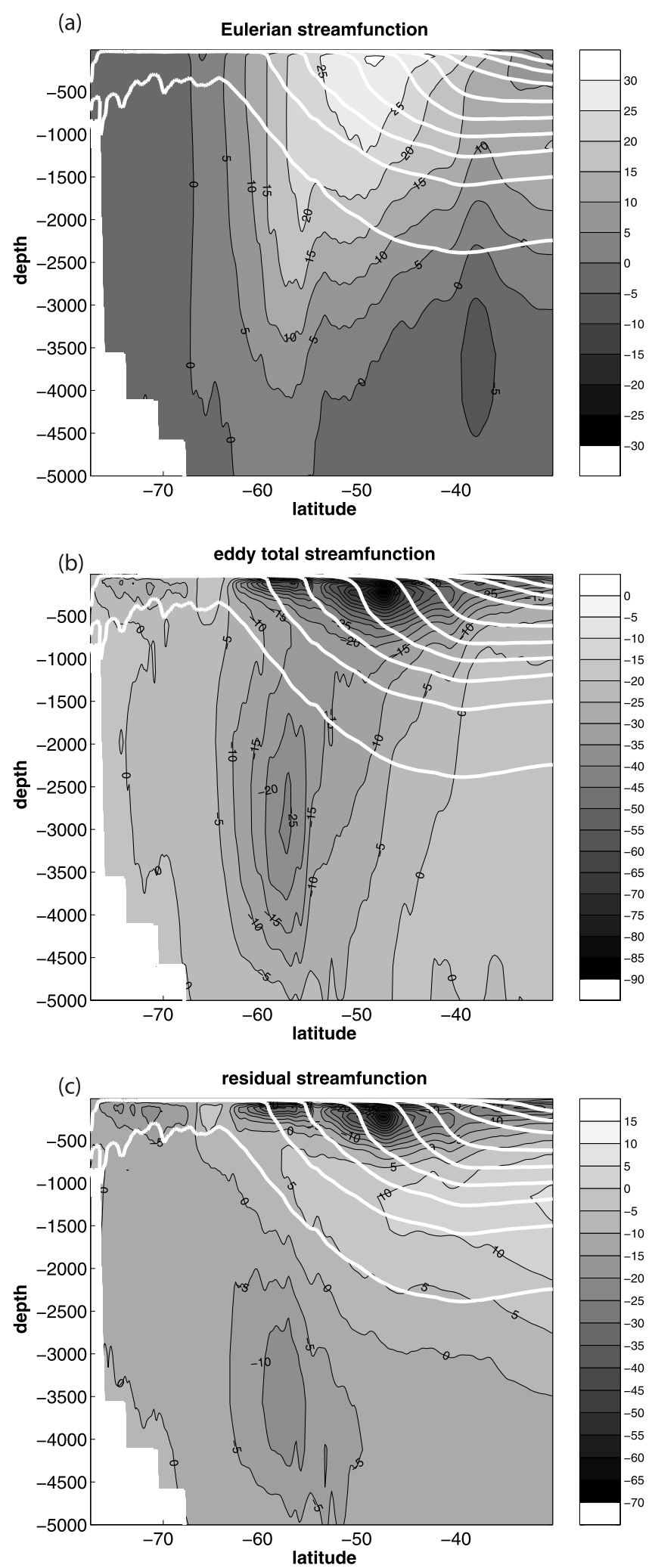

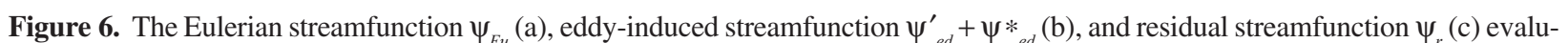
ated from the POP model. The white fat lines represent isopycnals. The units are Sv; CI = 5 Sv [from Olbers and Ivchenko, 2001]. 
For the transient eddies,

$$
{\overline{\mathbf{u}^{\prime} \rho^{\prime}}}^{x t}=-K_{d}^{\prime} \nabla \bar{\rho}^{x t}-\psi_{e d}^{\prime} \nabla_{s} \bar{\rho}^{x t},
$$

with $\mathbf{u}=(v, w), \nabla=(\partial / \partial y, \partial / \partial z)$, and $\nabla_{s}=(-\partial / \partial z, \partial / \partial y)$. Forming the divergence of this expression reveals that $K_{d}^{\prime}$ is an eddy-induced diapycnal diffusivity (if positive), and $\Psi_{\text {ed }}^{\prime}$ is an eddy streamfunction advecting the density $\overline{\rho(y, z)}$ in addition to $\Psi_{\mathrm{Eu}}$. A corresponding separation can be made for the standing eddies, denoted by the star below, but it is obvious that the construction of a diapycnal diffusivity for this type of eddies is less meaningful. Nevertheless, the advection of $\bar{\rho}^{x t}$ is achieved by the residual streamfunction

$$
\psi_{r}=\psi_{E u}+\psi_{e d}^{\prime}+\psi_{e d}^{*},
$$

and the diapycnal transport represented by $K_{d}=K_{d}^{\prime}+K_{d^{\prime}}^{*}$ All three components of the residual streamfunction $\psi_{r}$ play an important role in the ACC area. If we discard the eddy components and keep only the Eulerian component, we find a strong Deacon Cell. Taking all three components drastically reduces it.

We should mention that the definition of the TEM streamfunction and diffusivity as given by (18) is not unique. Simpler (but less canonical) forms have been discussed [Andrews and McIntyre, 1976; Held and Schneider, 1999] and used in the definition (15) and (16) of the residual circulation. These forms partly suffer from incorrect boundary conditions or the a priori assumption of the adiabatic nature of the flow. Indeed, assuming adiabatic conditions, $K_{d}^{\prime}=0$, we find $\psi_{e d}^{\prime}=\frac{{\overline{v^{\prime} \rho^{\prime}}}^{x t}}{{\overline{\rho_{z}}}^{x t}}$. More general than (18) is the additional consideration of a rotational flux term which may be used to gauge the eddy streamfunction and diapycnal diffusivity [Eden et al., 2007].

The highest values of transient eddy-induced meridional velocity occur between $65^{\circ} \mathrm{S}$ and $40^{\circ} \mathrm{S}$. This high value is related to the ACC area and strong baroclinic or (and) barotropic instability of the current. The distribution is of a dipole type in the POP model [Olbers and Ivchenko, 2001], with the largest values concentrated around two centers. The first cell is located in the upper $200 \mathrm{~m}$, and the second one is centered at about $2,800 \mathrm{~m}$, while its vertical range is between 1,000 and 4,000 m, with maximum transport of $30 \mathrm{~Sv}$. The latter cell mainly appears because of the input from the Southeastern Pacific where the EKE is greatest. These high values are observed in the vicinity of the main core of the ACC because of enhanced instability.

A large component of the standing eddy-induced streamfunction appears in the upper $500 \mathrm{~m}$, which strongly in- creases the total eddy-induced streamfunction (sum of transient and standing; see Figure 6b). This arises from diabatic effects, strong meridional excursions of the main core of the $\mathrm{ACC}$ in the area to the north of the ACCB, and also because a rotational part of the eddy fluxes has not been considered. The meridional streamfunction $\psi_{r}$ in the ACCB and below $500 \mathrm{~m}$ is more aligned with the zonal mean isopycnals compared to $\psi_{E u}$ (Figure 6c). With streamwise averaging, the standing contributions are largely absent.

Hallberg and Gnanadesikan [2006] find two ways the eddies affect the overturning in the Southern Ocean. First, they substantially extend the southward transport of relatively light water across the ACC [see also Drijfhout, 2005]. Second, the response to changing wind stresses is systematically smaller than in models with parameterized eddies. The response of the overturning circulation is concentrated on shallower isopycnals (layers).

Recognizing the role played by eddies in setting the meridional circulation, Marshall and Radko [2003], Olbers and Visbeck [2005], and Radko and Marshall [2006] propose simple models to estimate the streamline-averaged residual circulations and the density structure of the ACC. Gallego et al. [2004] pursue a similar goal based on a two-layer model. The basic assumption here is the absence of diapycnal fluxes below the mixed layer, in which case, the "zonally" averaged density equation reduces to

$$
J\left(\psi_{r}, \bar{\rho}^{x t}\right)=0 .
$$

Here, $J$ denotes the Jacobian operator. This equation implies that the residual streamfunction is a function of only mean density, $\psi_{r}=F\left(\bar{\rho}^{x t}\right)$, which is constant on streamlines of the residual velocity [characteristics of the differential equation (20)]. Knowing the density and the functional relation between it and $\psi_{r}$ just below the mixed layer, the buoyancy structure in the thermocline can be recovered [Marshall and Radko, 2003].

Olbers and Visbeck [2005] go a step further. They incorporate a model of mixed layer physics and link the slope of isopycnals on the base of the mixed layer to the surface forcing (wind stress and surface buoyancy flux). They then integrate (20) along characteristics to obtain the mean density structure. Radko and Marshall [2006] try to add the gravest mode of zonal variations to this approach.

Common to all these approaches is the parameterization of the eddy-induced velocity in terms of the isopycnal slope (essentially the Gent-McWilliams [1990] parameterization), and the assumption that the flow is adiabatic below the mixed layer. Olbers and Visbeck [2005] show that given a realistic surface forcing, a realistic density structure can be recovered for the Southern Ocean. These models, although extremely simplified and limited to characteristics exiting from the 
base of the mixed layer, do emphasize the contribution from eddies to the meridional overturning and propose estimates of eddy-induced and residual circulations.

The total transport of substance in an isopycnal layer consists of the transport by residual velocity (i.e., sum of Eulerian and eddy-induced) and eddy diffusion (see (18), (19), and Lee et al. [2007]). The direction of eddy advective transport is governed by the large-scale cross-isopycnal vertical tracer gradient, and the eddy diffusive transport is governed by the large-scale along-isopycnal meridional tracer gradient [see Lee et al., 2007]. They also show that the eddy advective and diffusive heat transports in the Southern Ocean are both poleward in OCCAM $\left(1 / 12^{\circ}\right.$ model). In contrast, eddy advective and diffusive salt transports are equatorward and poleward, respectively. Their results are consistent with those of Stammer [1998], where eddy heat and salt transport are inferred from assimilating satellite altimetry and other data. Henning and Vallis [2005] show by using a primitive equation high-resolution model with idealized geometry that the residual flow is balanced mainly by the cross-isopycnal eddy flux convergence in the channel region. Eddy buoyancy and heat fluxes can play an important role in the formation of water masses, particularly intermediate waters, by exchanging water between subtropical gyres and the ACC [Schouten and Matano, 2006].

\section{ENERGY BALANCES AND INSTABILITY}

The eddy field simulated in numerical models is a good indicator of model skill, as a growing amount of measurement data is becoming available through the progress in satellite altimetry and new measurements with subsurface floats. The performance of models in this respect is a special issue for the Southern Ocean where eddy contribution is playing a decisive role in meridional transports. The basic questions are:

-What is the mean level of the EKE?

-What is the horizontal and vertical distribution of the EKE?

-What is the main instability mechanism in the Southern Ocean? It is important to have a clear view on the instability processes in models to assess to what extent they are eddy-resolving and whether a further increase in resolution is needed before the Southern Ocean can be modeled realistically. As the Southern Ocean is very inhomogeneous with respect to the EKE, such an assessment can only be made on a regional basis [Ivchenko et al., 1997b].

-What are the main terms in the energy budgets? This question is closely related to the previous one. One can estimate the energy content and energy transfers directly from the model output.
An equation for the EKE can easily be derived from the equation of motion and can be written in the following form:

$$
\begin{aligned}
& \frac{\partial}{\partial t}\left(\frac{{\overline{u_{m}^{\prime 2}}}^{t}}{2}\right)=0=-\frac{\partial}{\partial x_{j}}\left({\overline{u_{j}}}^{t} \frac{{\overline{u_{m}^{\prime 2}}}^{t}}{2}\right)
\end{aligned}
$$

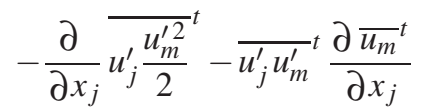

$$
\begin{aligned}
& -\frac{1}{\rho_{0}} \frac{\partial{\overline{u_{j}^{\prime} p^{\prime}}}^{t}}{\partial x_{j}}-\rho_{0}^{-1} g{\overline{u_{3}^{\prime} \rho^{\prime}}}^{t}+W_{H}^{\prime}+W_{Z}^{\prime},
\end{aligned}
$$

where the index $m=1,2$ represents the horizontal directions, while the index $j=1,2,3$ represents both horizontal and vertical $(j=3)$ directions; summation over the repeated indices is implied; $W_{H}^{\prime}$ and $W_{Z}^{\prime}$ represent the viscous terms (horizontal and vertical, respectively) and $\overline{u_{m}^{\prime \prime}} / 2$ is the EKE.

The first two terms on the rhs of (21) are the advection of the EKE by mean and eddy flows, respectively; the third term is the Reynolds stress work. The same term, but with the opposite sign appears in the balance of the kinetic energy of the mean flow, and therefore, it represents the exchange between mean and eddy kinetic energy. The forth term is the work by eddy pressure flux; the fifth term represents an exchange between eddy kinetic and potential energy. Note that the fourth and fifth terms are the parts of the eddy pressure

$$
\begin{aligned}
\text { work }(\mathrm{PW})^{\prime} & =-\frac{1}{\rho_{0}}{\overline{u_{m}^{\prime}}}^{\frac{\partial p^{\prime}}{\partial x_{m}}}: \\
(\mathrm{PW})^{\prime} & =-\frac{1}{\rho_{0}} \frac{\partial{\overline{u_{j}^{\prime} p^{\prime}}}^{t}}{\partial x_{j}}-\frac{g}{\rho_{0}}{\overline{u_{3}^{\prime} \rho^{\prime}}}^{t} .
\end{aligned}
$$

The first, second, and fourth terms on the rhs of (21) are written in a divergent form, which means that they redistribute the EKE (first and second) and eddy component of pressure work (fourth) inside the basin. They disappear after integration over a closed basin in a rigid lid approach.

After integration over the volume of a subregion with possible open boundaries, equation (21) becomes:

$$
N^{\prime}+\Pi^{\prime}+B^{\prime}+F_{H}^{\prime}+F_{Z}^{\prime}=0,
$$

where $N^{\prime}$ is the nonlinear term, representing the fluxes of the EKE through the open boundaries by the mean flow and eddies, and the Reynolds stress work; $\Pi^{\prime}=-\rho_{0}^{-1} \int_{(A)} \overline{u_{j}^{\prime} p^{\prime}} t d A_{j}$ is the eddy pressure flux term through boundaries; $B^{\prime}=$ $-\int_{(V)} \rho_{0}^{-1} g \overline{u_{3}^{\prime} \rho^{\prime}} d V$ is the buoyancy term; $F_{H}^{\prime}=\int_{(V)} W_{H}^{\prime} d V$ is an 
integral over volume $V$ of the horizontal friction, and $F_{z}^{\prime}$ is an integral over volume $V$ of the vertical friction. The nonlinear term $N^{\prime}$ can be written as:

$$
\begin{aligned}
N^{\prime}= & -\int_{(A)}\left({\overline{u_{j}}}^{t} \frac{{\overline{u_{m}^{\prime 2}}}^{t}}{2}+{\overline{u_{j}^{\prime}}}_{\frac{u_{m}^{\prime 2}}{2}}^{t}\right) d A_{j} \\
& -\int_{(V)}{\overline{u_{j}^{\prime}}}^{t}{ }^{t} \frac{\partial{\overline{u_{m}}}^{t}}{\partial x_{j}} d V,
\end{aligned}
$$

where $A$ is the boundary of the subregion, and $d A_{j}$ is the oriented surface element normal to this boundary.

The EKE budget for the ACC area from the FRAM experiment shows a balance between the two biggest terms, the generation of the EKE by buoyancy $B^{\prime}$, and the horizontal friction $F_{H}^{\prime}$ [Ivchenko et al., 1997b]. Observations also give support to the importance of the dissipation of eddies in the circumpolar region [Bryden, 1983]. The other terms, that is, nonlinear transfers, pressure flux through the open boundary, and bottom friction are much smaller. The EKE budget for a wider area of the Southern Ocean from $27.5^{\circ} \mathrm{S}$ to the Antarctic coast (nearly all FRAM area) is substantially different: The main sources are both the buoyancy term $B^{\prime}$ and the nonlinear transfer term $N^{\prime}$. These two sources are mainly balanced by the lateral friction. The importance of lateral friction arises because several gyres and western boundary currents are included in the domain. High velocities and horizontal velocity shears are observed there. The buoyancy term $B^{\prime}$ is most important in the ACC. If we consider the FRAM regions where $B^{\prime}$ is a source of EKE, $92 \%$ of generation is confined to the ACC [Ivchenko et al., 1997b]. When the ACC is split into the ACCB and the North ACC domain (the area to the north of the ACCB), the ACCB contributes $24 \%$ and the North ACC contributes $76 \%$ of the total. This shows that an analysis restricted to the ACCB region misses the largest part of the eddy activity in the ACC.

The ratio $\alpha=N^{\prime} / B^{\prime}$ is much higher in the gyre-type domain than in the channel-type domain [Ivchenko et al., 1997b]. For the whole FRAM domain $\alpha=0.6$, which is to be compared to $0.04,0.02$, and 0.02 for the ACCB, the ACC part outside the Drake Passage latitudes and the whole ACC, respectively. Note that in other models in such regions as the Northern Atlantic, $\alpha$ is often even greater than 1 .

The regional analysis of energy budgets and instability could complement the zonal and streamwise analysis. The strongest values of the EKE correspond to the most pronounced topographic features in the Southern Ocean. Measuring the eddy fluxes should be focused on areas downstream of the topography [Best et al., 1999; Hallberg and Gnanadesikan, 2001]. The nonlinear transfer $N^{\prime}$ is a source of EKE in many subregions in the FRAM. It may be interpreted as a conversion from the kinetic energy of mean flow to EKE and can be related to the barotropic instability. This conversion is toward the EKE in most regions in the FRAM.

The baroclinic instability can be diagnosed by computing the $A^{\prime}$ term (introduced by Böning and Budich [1992] and not given here), which is approximately the exchange between potential energy of the mean flow and the eddy potential energy. This term is proportional to the horizontal eddy density flux multiplied by the mean horizontal density gradient and inversely proportional to the vertical gradient of the potential density of the reference state. In the ACC area $A^{\prime}$ and $B^{\prime}$ have the same signs and are of the same magnitude. This is consistent with the classical picture of baroclinic instability in which eddy potential and kinetic energies are created out of the potential energy of the mean flow. The ratio of $B^{\prime}$ of the North ACC to that of the ACCB region is almost equal to the ratio of their EKE densities [Ivchenko et $a l ., 1997 \mathrm{~b}]$. This further suggests that the energy levels in these regions are closely linked to the strength of baroclinic instability occurring in each region. For the Antarctic Zone subregion (between the southern boundary of the ACCB and the Antarctic coast), $B^{\prime}$ is larger than $A^{\prime}$, which clearly means that the source of EKE is not linked completely to baroclinic instability. Furthermore, the FRAM horizontal grid does not resolve the eddies in this subregion because of weak stratification resulting in a small Rossby radius.

Baroclinic instability can be examined by calculating unstable modes of the zonal flow. The eigenvalue problem is solved in a manner described by Beckmann [1988] for the spatially and time-averaged shear of zonal flow and the corresponding mean density profile. The analysis was applied to a number of dynamically important subregions both inside and outside the ACC based on the FRAM and POP model outputs [Best et al., 1999; Wells et al., 2000]. In all the regions considered, the flow has been found to be baroclinically unstable. In FRAM, the growth rate, that is, the e-folding time of the baroclinic instability, ranges from 3 to 65 days, and in the POP, from 8 to 312 days. In the majority of regions, the most unstable wavelengths are marginally resolved by the zonal grid spacing. Maximum growth rates are found to occur on scales from approximately 1.6 to 3.6 times the first Rossby radius in the FRAM and from 1.3 to 7.1 times the first Rossby radius in the POP analysis.

Instability analysis performed over the ACC jets in the FRAM and POP models showed that baroclinic instability is likely to be the main route for generating EKE. Barotropic instability also contributes across several localized places with especially strong mean velocities and horizontal velocity shears. For example, barotropic instability develops between the Agulhas and Agulhas Return Current [Wells et al., 
2000]. On the northern flank of the Agulhas Current, there is an upgradient momentum flux into the mean flow.

In the FRAM, the upstream flows and flows just to the northeast of the Drake Passage have similar growth rates that are reasonably fast (24 days). A significant difference between the two regions is that the EKE density in the upstream flow is almost six times higher than that of the northeastern flow (58 and $10 \mathrm{~cm}^{2} \mathrm{~s}^{-2}$, respectively). One reason why the flow northeast of Drake Passage remains relatively stable in the FRAM, despite the predicted instability, is that the wavelength at which instability is most likely to occur is only resolved by three zonal grid points. Furthermore, the local first baroclinic Rossby radius is only just over one grid box in length. This strongly suggests that the stability of the flow downstream of the Drake Passage is due to the lack of resolution in FRAM. By way of contrast, the POP gives vigorous eddy distributions downstream of Drake Passage: The EKE density is 39 and $129 \mathrm{~cm}^{2} \mathrm{~s}^{-2}$ for the upstream and northeast regions, respectively. Similar vigorous eddy distribution is clearly seen in the TOPEX data in the northeast region. The instability analysis of the POP model in this region, however, gives a very slow growth rate of 312 days. One possible explanation for this is that downstream of Drake Passage barotropic instability is the dominant mechanism. This may be expected since the jet produced by the POP is very tight.

\section{ZONAL JETS}

The ACC is seen as a broad current in coarse-resolution numerical models. With increasing resolution, the oceanic general circulation models (OGCM) are gaining skill in presenting separate jets and the associated frontal structure as illustrated in Plate 1 [adopted from Hallberg and Gnanadesikan, 2006; also see Maltrud et al., 1998; Sinha and Richards, 1999; Richards et al., 2006]. The major fronts in the ACC include the Subantarctic, Polar, and the Southern ACC fronts; yet, a finer frontal structure can be distinguished by closer inspection. Based on the analysis of hydrography at $140^{\circ} \mathrm{E}$ in the ACC, Sokolov and Rintoul [2002] show that the major fronts are split in reality in several branches. There is still some controversy with respect to precise positions of the major fronts [see Hughes and Ash, 2001], and even their circumpolar character is not easy to prove in all sectors of the Southern Ocean. The fine frontal structure is variable in time; the jets may appear and disappear by coalescing with each other, and some of them exist only locally. The major fronts are separating water masses with distinct properties. The fine frontal structure is seemingly of dynamic origin.

Despite the progress achieved recently with the fineresolution OGCM in modeling the Southern Ocean, simulat- ing the observed fine frontal structure still awaits for models with better spatial resolution and perhaps also constrained with observation data. The physical mechanisms of jet formation and the role of eddies in maintaining the jets are not fully understood, and existing evidence is rather controversial [see Hughes and Ash, 2001]. The frontal (jet) structure of the ACC is frequently explained by invoking arguments of $\beta$-plane turbulence [Rhines, 1975]. Indeed, the prediction of jet formation on the Rhines scale agrees generally well with results found in the QG layer models, demonstrating the appearance of multiple jets due to eddies generated by baroclinic instability [see, e.g., Panetta, 1993; Treguier and Panetta, 1994; Sinha and Richards, 1999]. The jet formation or sharpening is linked to the eddy convergence of eastward momentum, and a number of QG studies provide a nice illustration of this fact [Wolff et al., 1991; Panetta, 1993; Treguier and Panetta, 1994; Olbers, 2005].

The real situation is much more complicated because bottom topography leads to strong localization of jets downstream of the major topographic features in the Southern Ocean, which influences jet formation and spacing. The simple argument suggested by the theory of two-dimensional turbulence is not necessarily working everywhere in the ACC, yet still remains a plausible departure point.

In this work, we pursue a modest goal of presenting an elementary view on a jet formation mechanism in $\beta$-plane turbulence and confronting it with results that follow from existing modeling efforts.

\subsection{Phenomenological View on Barotropic $\beta$-Plane Turbulence}

We begin with barotropic $\beta$-plane turbulence driven by small-scale forcing in a flat-bottom box. This is the most elementary system capable of producing multiple jets due to turbulent eddies. It might shed some light on jet formation in the ACC because of its equivalently barotropic character [Killworth, 1992; Killworth and Hughes, 2002]. According to Rhines [1975], barotropic turbulence on a $\beta$-plane tends to form a jet-like structure with wavenumber

$$
k_{R h}=(\beta / 2 U)^{1 / 2},
$$

which is simultaneously the scale where the energy cascade toward large scales is arrested. Here, $U$ is the eddy rms velocity. The physical explanation for this tendency is the presence of Rossby wave dispersion that reduces the efficiency of nonlinear transfer involving quasi-zonal wavevectors so that the turbulent energy concentrates at the meridional wavenumber $k_{R h}$. Numerous simulations with barotropic models [Vallis and Maltrud, 1993; Danilov and Gurarie, 2002; 
Smith et al., 2002] suggest that the Rhines scale performs well in predicting the observed scale $k_{\mathrm{obs}}$ of jets, with a factor $k_{\mathrm{obs}} / k_{\mathrm{Rh}}$ being between 0.7 and 1.5 in most cases. Similar skill is also seen in layered QG models [Sinha and Richards, 1999]. Taking a midlatitude estimate for $\beta=1.5 \times 10^{-11} \mathrm{~m}^{-1}$ $\mathrm{s}^{-1}$ and an $\mathrm{rms}$ velocity of $10 \mathrm{~cm} / \mathrm{s}$, one gets a wavelength of $700 \mathrm{~km}$, which is too large compared to the actual distance between the fronts observed in the ACC [see Sokolov and Rintoul, 2002]. The influence of topography and baroclinicity can be responsible for this discrepancy [Sinha and Richards, 1999].

For a flat-bottom barotropic flow, the Rhines scale can easily be recast in terms of energy production rate $\varepsilon$. If the flow is stabilized at large scales by bottom drag with the inverse timescale $\lambda$, the total eddy kinetic energy is then $E=$ $\varepsilon / 2 \lambda$, giving

$$
k_{R h}=\beta^{1 / 2}(\lambda / 4 \varepsilon)^{1 / 4} .
$$

This estimate emphasizes the roles of forcing and dissipation and allows answering the question why zonation is not always observed even if eddies are present.

If the $\beta$ effect were absent, the inverse energy cascade is stopped by the bottom drag on the friction scale $k_{f r}=\left(3 C_{K}\right)^{3 / 2}$ $\left(\lambda^{3} / \varepsilon\right)^{1 / 2}$ (with $\left.C_{K} \approx 6\right)$. Jets are formed only if $k_{R h}$ exceeds $k_{f r}$ because otherwise, the inverse cascade is arrested before it reaches scales where the Rossby dispersion is important [Danilov and Gurarie, 2002; Smith et al., 2002]. The ratio $\gamma=k_{R h} / k_{f r}=A \beta^{1 / 2} \varepsilon^{1 / 4} \lambda^{-5 / 4}=A \beta^{1 / 2} U^{1 / 2} / \lambda$ defines the boundary between the regimes without $(\gamma<1)$ and with $(\gamma>1)$ jets (here, $\left.A=4^{-1 / 4}\left(3 C_{K}\right)^{-3 / 2}\right)$. For the ACC taking $\lambda=0.01$ day $^{-1}$, one gets $\gamma \approx 0.12\left(U=10 \mathrm{~cm} \mathrm{~s}^{-1}\right)$, which is too low and no jets are expected. But if $\lambda$ is reduced to 0.0015 day $^{-1}, \gamma$ approaches 1 , and jets will be produced by small-scale stirring in a barotropic flow.

Clearly, the role of friction is overemphasized in the barotropic approach, and the $\gamma$ scaling can be applied to the ACC only qualitatively. Yet, it draws attention to the impact of dissipation on the jet formation. Many jets are local features that do not continue over the entire Southern Ocean. The strength of eddies can plausibly depend on the local energy balance between forcing and dissipation. The ability of models to reproduce the jets then depends on their level of dissipation.

Large-scale topography influences barotropic turbulence in an obvious way by redefining the local gradient of unperturbed QPV from $\beta$ to a combination of $\beta \mathbf{j}+f_{0} \nabla b / H_{0}$, where $H_{0}$ is the total depth. The second component adds with $\beta$ on topographic features sloping southwards (in the southern hemisphere) and can easily dominate locally. In such situations, the increase in effective $\beta$ reduces jet spacing. A slope of 0.001 reduces the wavelength by a factor of around two when its effect adds with $\beta$. Sinha and Richards [1999] show that taking the topographic slope into account does indeed make the jet spacing observed in the FRAM and POP consistent with theory if the Rhines scale is computed with the mean effective $\beta$.

The phenomenology of $\beta$ plane turbulence leaves obscure the mechanism of jet formation. It turns out that this mechanism can be understood from a kinematic viewpoint involving the concept of PV mixing by eddies. This concept was used by Danilov and Gryanik [2004] and is elaborated from a broader physical perspective by Dritschel et al. [2008].

\subsection{Multiple Jets From a Kinematic Perspective}

Consider first a barotropic flow created by a small-scale stirring within a relatively narrow latitude belt $y_{1} \leq y \leq y_{2}$ on a $\beta$ plane. The Rossby waves will be generated there and, if friction is moderate, dissipated mostly outside the region of generation. The outgoing Rossby waves are associated with the divergence of the eastward momentum flux and on dissipation leave westward flows outside the stirring belt. If stirring does not impart mean momentum (as is the case with eddies produced by baroclinic instability), a compensating eastward flow occurs in the source region, a situation resembling that at midlatitudes in the atmosphere [see also Rhines, 1994; Vallis, 2006].

This reasoning is the simplest way of linking the generation of outgoing Rossby waves to the convergence of eastward momentum flux in the stirring region. When a mean background current is present, the Rossby waves are modified, and the waves are existing due to the mean PV gradient. This is assumed everywhere in this section.

Now let us look at this flow pattern from a PV perspective (we assume QG scaling and deal with QPV). Formation of westward flow north of $y_{2}$ and eastward flow in some vicinity south of it implies PV mixing around $y_{2}$ that tends to locally reduce the QPV gradient there. Mixing is not necessarily perfect and may leave a residual QPV gradient. The same argument applies to the southern source boundary $y_{1}$. One gets two zones of partly mixed QPV on flanks of the eastward jet. This mixing is irreversible, as it accompanies dissipation of Rossby waves.

There should be a QPV front joining the zones of mixed QPV within the source region that defines the eastward jet. Thus, the irreversible PV mixing due to Rossby wave dissipation is conductive to formation of PV fronts and eastward jets within the stirring belt. Clearly, this is simply a different language for expressing the well-known phenomena of eastward momentum flux convergence associated with eastward jets (see section 2.1).

If the stirring belt is sufficiently wide, several QPV fronts may form and coexist simultaneously within it, separating 
zones of partly mixed QPV. The stability of fronts depends on their strength (the QPV jump across the front). Indeed, for a fluid particle to pass across the front, its QPV should be modified by the QPV jump amplitude. Thus, fronts of small amplitude will be destroyed by stirring that leaves wider zones of mixed QPV limited by QPV fronts with higher QPV jumps. Fronts are becoming stronger if zones of mixed QPV are becoming wider (for a fixed degree of mixing). Full mixing is never achieved in practice, as it eliminates the Rossby waves required to redistribute QPV (or momentum) in the meridional direction.

For a given degree of PV mixing, the kinetic energy per unit mass of a zonal flow associated with the mixed zone increases with its width $l$ as $l^{4}$. The dissipation of kinetic energy grows accordingly. Yet, it should be balanced with energy generation. This balance sets the size of the zone of mixed QPV, which is clearly the Rhines scale up to a factor of order one.

A limiting case of this scenario is what happens if smallscale stirring is uniform over the entire $\beta$ plane. The result is a staircase structure of partly mixed QPV zones (or a comblike structure of the QPV gradient similar to that of Panetta [1993]). Writing the gradient of relative vorticity within a zone as $-a \beta(0 \leq a \leq 1)$ and requiring the total momentum be zero, one finds the zonal velocity profile

$$
u=0.5 a \beta\left(\left(y-y_{c}\right)^{2}-l^{2} / 12\right)
$$

within a zone of width $l$ centered at $y_{c}$. Expressing the kinetic energy, one obtains $k_{R h} l \approx 3.7 / a^{1 / 2}$. This implies that the Rhines scaling is in essence the consequence of QPV mixing in zones between two neighboring fronts, and the success of this scaling in predicting the distance between the jets depends on the degree of mixing inside the zone $a$.

This view translates to the general case of a baroclinic flow having, like the ACC, a wide zone of high baroclinicity. The interpretation is straightforward for layered flows, and indeed, the PV structure of their upper layers does support the concept of PV mixing (see Figure 4 of Panetta [1993] showing a comblike structure for the upper layer QPV gradient and Figure 4a of MacCready and Rhines [2001] displaying bands of mixed PV in the upper layer downstream of the topographic ridge). For a continuously stratified fluid, it is the sign of surface or bottom buoyancy gradients that typically determines the baroclinic instability (alternatively, one may introduce surface and bottom QPV sheets). The PV mixing concept then also involves fronts and mixed zones in the surface or bottom buoyancy.

Summing up, the irreversible PV mixing mediated by Rossby waves is a mechanism for multiple jet formation.

\subsection{Confronting Theory and Observations}

Can the jets in the ACC be explained by the simple theory of QG turbulence? The answer is that it is only partly applicable, and there are two main issues to be mentioned.

First, although Sinha and Richards [1999] show that there is a good agreement (on average) between the observed and predicted jet spacing in the FRAM and POP models, closer inspection reveals a difficulty. Figure 7 reproduces their result showing mean zonal velocity and bottom topography at several longitudinal locations in the ACC as derived from the output of the FRAM (left column) and the POP model (right column). Jet spacing is smaller above the southern slope of the ridge centered at $50^{\circ} \mathrm{S}$ (where effective $\beta$ is small) than above the northern slope in the upper row, and there is no significant difference in spacing above southern and northern slopes in the lower two panels of Figure 7 (but effective $\beta$ differs considerably). Therefore, using a local value of effective $\beta$ would lead to disagreement between observations and theory.

Richards et al. [2006] present a wavelet power spectrum of the zonal component of velocity in the Pacific sector of the ACC simulated by the POP model at $1 / 10^{\circ}$ resolution. They mention discrepancy between the observed scale and the Rhines scale (about a factor of 2).

Clearly, the comparison of observed (simulated) jet scale with the Rhines scale is somewhat ambiguous because no unique sense can be attached to $U$ in a baroclinic flow. Yet, the power spectra for the equatorial belt and the belt of latitudes in the Northern Pacific presented by Richards et al. [2006] demonstrate much closer coincidence between the model and theory which supports the view that their estimate for $U$ is consistent.

The observational evidence is also in favor of the possibility of relatively small distances between the jets. Sokolov and Rintoul [2002], for example, show the pattern of the sea surface height contours in the $130^{\circ}-160^{\circ} \mathrm{E}$ sector that coincide with the fronts identified at section SR3, which are in some places about $1^{\circ}$ apart. Even finer frontal structure is indentified by Sokolov and Rintoul [2007] based on the analysis of satellite altimetry data. Such a separation between fronts is somewhat too small to be explained by QG turbulence theory (unless the amplitude of $U$ is essentially smaller than the $10 \mathrm{~cm} / \mathrm{s}$ used here for estimates).

Second, the analyses by MacCready and Rhines [2001] based on a two-layer isopycnal model and by Hughes and Ash [2001] based on satellite-derived surface geostrophic velocities and eddy fluxes both reveal that eddies do not necessarily accelerate the jets, contrary to the theoretical predictions. Similarly, in the FRAM [Ivchenko et al., 1997b] and the POP model [Best et al., 1999], the integrated effect of eddies is to decelerate the flow. 

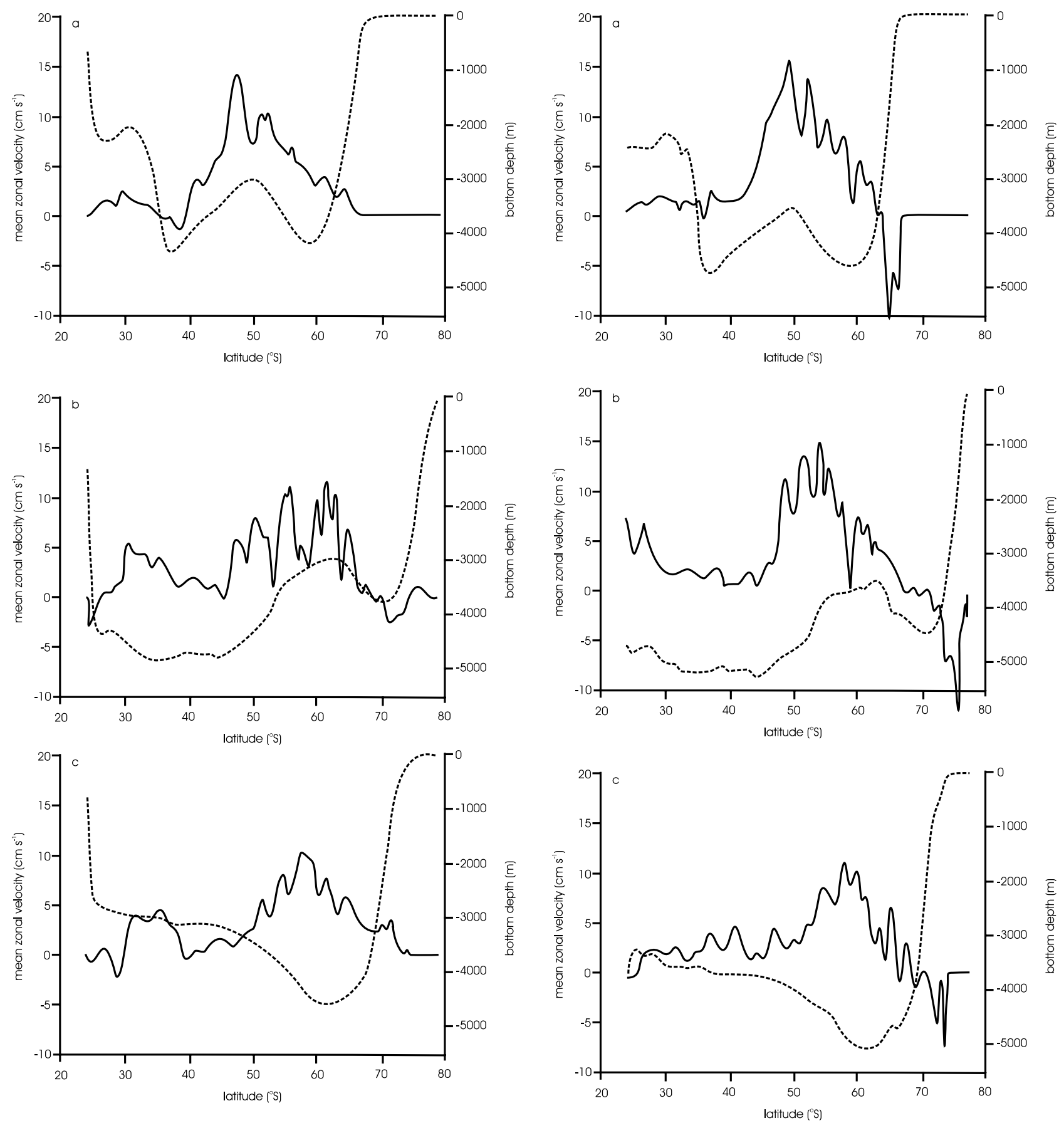

Figure 7. Longitude averaged time mean near-surface $(32.5 \mathrm{~m}$ ) zonal velocity for the FRAM (left column) and POP (right column) models (solid line) for (a) $85^{\circ}-135^{\circ} \mathrm{E}$, (b) $190^{\circ}-225^{\circ} \mathrm{E}$, and (c) $240^{\circ}-280^{\circ} \mathrm{E}$. The dashed lines represent the bottom topography averaged along longitude [from Sinha and Richards, 1999].

MacCready and Rhines [2001] consider a flow in a zonally reentrant channel with a topographic ridge and show that the eddy Reynolds stress divergence acts to decelerate jets along lines of constant pressure. Locally, there are regions of acceleration and deceleration, with the latter dominating. However, zonal averaging reveals narrow regions 
around centers of major jets where the contribution from the Reynolds stress divergence is accelerating. Yet, over the downstream part of the slope where the current is strongest, the contribution from the Reynolds stress divergence is to slow and broaden the jets.

Hughes and Ash [2001] computed the rotational part of the Reynolds stress divergence by processing surface geostrophic velocities derived from TOPEX/Poseidon and ERS altimetry. Their computations show that eddies act to decelerate the jets past main topographic features or act as vorticity sources for other strong jets, consistent with the observation by MacCready and Rhines [2001]. However, the sign of eddy force is less clear away from the major topographic features, and one can distinguish contributions of both signs there.

As Hughes and Ash [2001] point out, the key factor in explaining this behavior seems to be the bottom topography, which can tend to produce very narrow jets by topographic steering. Broadening of such narrow jets due to eddy stirring is to be expected past topography, and it is equivalent to jet deceleration by eddies.

In flat-bottom experiments, it is only the eddy forcing that maintains narrow jets against dissipation by a mechanism linked to PV mixing. The jets and eddies are in quasiequilibrium at every zonal location because flow is statistically "invariant" along a zonal coordinate. In the presence of topography, the jet shape, spacing, and baroclinicity of the flow are strongly modified as the jet passes a topographic feature. One can speculate that equilibrium between local generation of turbulence due to baroclinic instability and backward action of turbulent eddies on jets can only be reached further downstream or not reached at all if a new topographic obstacle is encountered. Accordingly, the Rhines scaling can only work qualitatively, while jet intensification by eddies should only be observed locally and presumably away from steep bottom topography. This answer, however, poses new questions as to how the interplay of topography and eddy forcing determines the fine frontal structure downstream of the main topographic features and what maintains this structure in relatively flat regions at scales that are seemingly too small. These questions require additional research and hopefully will be answered at some point in the future.

\section{SUMMARY AND DISCUSSION}

Substantial progress in understanding the basics of the Southern Ocean dynamics has been achieved during the last three decades. This understanding is linked to the advances in numerical ocean modeling and appearance of models resolving or at least permitting mesoscale eddies. The first step in this direction was made by using quasi-geostrophic models that allowed exploring basic balances, mechanisms of momentum penetration, and horizontal redistribution of momentum and energy [McWilliams et al., 1978; Treguier and McWilliams, 1990; Wolff et al., 1991]. However, the intrinsic limitations of these models prevented them from simulating reliable levels of energies, the ACC transport and its variability, as well as many other properties.

The second step involved eddy-resolving experiments carried out with primitive equation models. The output of several such models was employed in a number of studies to investigate the global and local balances mediated by eddies and bringing a quantitative flavor to the mechanisms through which eddies influence ACC dynamics. Substantial progress has been achieved in the studies of generation and propagation of water masses and tracers, in meridional heat transport, and atmospheric-ocean exchange.

There remain important questions concerning the ACC dynamics that are expected to be solved in the near future. They are directly or indirectly linked to eddies that are an indispensable part of the Southern Ocean physics. Theory of the frontal jets in the Southern Ocean, which can describe the fine structures downstream topographic features, is a new challenge for the community. A considerable effort in the modeling of the Southern Ocean is to go into studies of diabatic motion and its role in setting the residual circulation. The eddy dynamics in the Antarctic Zone (to the south of Antarctic Polar Front) is still relatively unexplored because of a weak stratification and a very small Rossby radius. Modeling the ice dynamics and thermodynamics with open boundaries and polynias and convective processes is also a challenge. Convection in the Southern Ocean can be a source of disturbances that quickly propagate from Antarctic to the equatorial ocean [Ivchenko et al., 2004, 2006; Richardson et al., 2005; Blaker et al., 2006]. New mechanisms of strong amplification of the waves coming to the equator were recently found [Reznik and Zeitlin, 2006]. The Antarctic sea ice distribution is strongly correlated with the NINO3 index of El Niño Southern Oscillation on timescales of an order of a few months [Yuan and Martinson, 2000; Kwok and Comiso, 2002]. Correlation occurs with both NINO3 and sea ice leading. Also important is the problem of teleconnections between the Antarctic and equatorial ocean. This problem can be discussed in the context of the role of the Southern Ocean in the global thermohaline circulation and global climate variability.

Acknowledgments. We are grateful to Bob Hallberg for permission to use the figure from Hallberg and Gnanadesikan [2006]. We thank M.-M. Lee for providing her new results, which are included within section 5 . 


\section{REFERENCES}

Andrews, D. G., and M. E. McIntyre (1976), Planetary waves in horizontal and vertical shear: The generalized Eliassen-Palm relation and the mean zonal acceleration, J. Atmos. Sci., 33, 2031-2048

Beckmann A. (1988), Vertical structure of mid-latitude mesoscale instabilities, J. Phys. Oceanogr., 18, 1354-1371.

Best S. E., V. O. Ivchenko, K. J. Richards, R. D. Smith, and R. C. Malone (1999), Eddies in numerical models of the ACC and their influence on the mean flow, J. Phys. Oceanogr., 29, 328-350.

Blaker, A. T., B. Sinha, V. O. Ivchenko, N. C. Wells, and V. B. Zalesny (2006), Identifying the roles of the ocean and atmosphere in creating a rapid equatorial response to a Southern Ocean anomaly, Geophys. Res. Lett., 33, L06720, doi:10.1029/ 2005GL025474.

Böning, C., and R. G. Budich (1992), Eddy dynamics in a primitive equation model: Sensitivity to horizontal resolution and friction, J. Phys. Oceanogr., 22, 361-381.

Bretherton, F. S. (1966), Critical layer instability in baroclinic flows, Q. J. R. Meteorol. Soc., 92, 325-334.

Bryden, H. L. (1983), The Southern Ocean, Chapter 14 in $E d-$ dies in Marine Science, edited by A.R. Robinson, pp. 265-277, Springer, Berlin.

Bryden, H. L., and S. A. Cunningham (2003), How wind-forcing and air-sea heat exchange determine the meridional temperature gradient and stratification for the Antarctic Circumpolar Current, J. Geophys. Res., 108(C8), 3275, doi:10.1029/2001JC001296.

Bryden, H. L., and R. A. Heath (1985), Energetic eddies at the northern edge of the Antarctic Circumpolar Current in the southwest Pacific, Prog. Oceanogr., 14, 65-87.

Danilov, S., and V. M. Gryanik (2004), Barotropic beta-plane turbulence in a regime with strong zonal jets revisited, J. Atmos. Sci., 61, 2283-2295.

Danilov, S., and D. Gurarie (2002), Rhines scale and spectra of the $\beta$-plane turbulence with bottom drag, Phys. Rev. E, 65, 067301.

Döös, K., and D. J. Webb (1994), The Deacon Cell and the other meridional cells in the Southern Ocean, J. Phys. Oceanogr., 24 429-442.

Drijfhout, S. S. (2005), What sets the surface eddy mass flux in the Southern Ocean? J. Phys. Oceanogr., 35, 2152-2166.

Dritschel, D. G., and M. E. McIntyre (2008), Multiple jets as PV staircases: The Phillips effect and resilience of eddy-transport barriers, J. Atmos. Sci., 65, 855-874.

Dukowicz, J. K., and R. D. Smith (1994), Implicit free-surface method for the Bryan-Cox-Semtner ocean model, J. Geophys. Res., 99(C4), 7991-8014.

Dukowicz, J. K., R. D. Smith, and R. C. Malone (1993), A reformulation and implementation of the Bryan-Cox-Semtner ocean model on the Connection Machine, J. Atmos. Oceanic Technol., 10, 196-208.

Eden, C., R. J. Greatbatch, and D. Olbers (2007), Interpreting eddy fluxes, J. Phys. Oceanogr., 37, 1282-1296.

Edmon, H. J., B. J. Hoskins, and M. E. McIntyre (1980), Eliassen-Palm cross sections for the troposphere, J. Atmos. Sci., 37, 2600-2616
Eliassen, A., and E. Palm (1961), On the transfer of energy in stationary mountain waves, Geophys. Publ., 22(3), 1-23.

Ferreira, D., J. Marshall, and P. Heimbach (2005), Estimating eddy stress by fitting dynamics to observations using a residual-mean ocean circulation model and its adjoint, J. Phys. Oceanogr., 35, 1891-1910.

Gallego, B., P. Cessi, and J. C. McWilliams (2004), The Antarctic Circumpolar Current in equilibrium, J. Phys. Oceanogr., 33, $1571-1587$

Gent, P, and J. C. McWilliams (1990), Isopycnal mixing in ocean circulation model, J. Phys. Oceanogr., 20, 150-155.

Gille, S. T. (1997), The Southern Ocean momentum balance: Evidence for topographic effects from numerical model output and altimeter data, J. Phys. Oceanogr., 27, 2219-2232.

Grezio A., N. C.Wells, V. O. Ivchenko, and B. A. de Cuevas (2005), Dynamical budgets in the Antarctic Circumpolar Current using ocean general circulation models, Q. J. R. Meteorol. Soc., 131, 833-860.

Hallberg, R., and A. Gnanadesikan (2001), An exploration of the role of transient eddies in determining the transport of a zonally reentrant current, J. Phys. Oceanogr., 31, 3312-3330.

Hallberg, R., and A. Gnanadesikan (2006), The role of eddies in determining the structure and response of the wind-driven southern hemisphere overturning: Results from the modelling eddies in the Southern Ocean (MESO) project. J. Phys. Oceanogr., 36 , 2232-2252.

Held, I.M. (1975), Momentum transport by quasi-geostrophic eddies, J. Atmos. Sci., 32, 1494-1497.

Held, I. M., and T. Schneider (1999), The surface branch of the mass transport circulation in the troposphere, J. Atmos. Sci., 56, 1688-1697.

Henning, C. C., and G. K. Vallis (2005), The effect of mesoscale eddies on the stratification and transport of an ocean with a circumpolar channel, J. Phys. Oceanogr., 35, 880-896.

Hughes, C. W., and E. R. Ash (2001), Eddy forcing of the mean flow in the Southern Ocean, J. Geophys. Res., 106, 2713-2722.

Ivchenko, V. O. (1987), The influence of bottom topography on the eddy transfer coefficient, Izvestiya Acad. Nauk USSR, Atmos. Ocean Phys., 21, 250-260.

Ivchenko, V. O., K. J. Richards, and D. P. Stevens (1996), The dynamics of the Antarctic Circumpolar Current, J. Phys. Oceanogr., 26, 753-774.

Ivchenko, V. O., K. J. Richards, B. Sinha, and J.-O. Wolff (1997a), Parameterization of mesoscale eddy fluxes in zonal ocean flows, J. Mar. Res., 55, 1127-1162.

Ivchenko, V. O., A. M. Treguier, and S. E. Best (1997b), A kinetic energy budget and internal instabilities in the Fine Resolution Antarctic Model, J. Phys. Oceanogr., 27, 5-22.

Ivchenko, V. O., V. B. Zalesny, and M. R. Drinkwater (2004), Can the equatorial ocean quickly respond to Antarctic sea ice/salinity anomalies? Geophys. Res. Lett., 31, L15310, doi:10.1029/ 2004GL020472.

Ivchenko V. O., V. B. Zalesny, M. R. Drinkwater, and J. Schröter (2006), A quick response of the equatorial ocean to Antarctic sea ice/salinity anomalies, J. Geophys. Res., 111, C10018,doi:10.1029/2005JC003061. 
Johnson, G. C., and H. L. Bryden (1989), On the size of the Antarctic Circumpolar Current, Deep Sea Res., 36, 39-53.

Kamenkovich, V. M., M. N. Koshlyakov, and A. S. Monin (1986), Synoptic Eddies in the Ocean, D. Reidel Publishing Company, Dordrecht, Holland, 433 pp.

Killworth, P. D. (1992), An equivalent-barotropic mode in the Fine Resolution Antarctic Model, J. Phys. Oceanogr., 22, 1379-1387.

Killworth, P. D., and C. W. Hughes (2002), The Antarctic Circumpolar Current as a free equivalent-barotropic jet, J. Mar. Res., 60, 19-45.

Killworth, P. K., and M. M. Nanneh (1994), On the isopycnal momentum budget of the Antarctic Circumpolar current in the Fine Resolution Antarctic Model, J. Phys. Oceanogr., 24, 1201-1223.

Kwok, R., and J. C. Comiso (2002), Southern Ocean climate and sea ice anomalies associated with the Southern Oscillation, $J$. Clim., 15, 487-501.

Lee, M.-M., and A. C. Coward (2003), Eddy mass transport for the Southern Ocean in an eddy-permitting global ocean model, Ocean Model., 5, 249-266.

Lee, M.-M., A. J. G. Nurser, A. C. Coward, and B. A. de Cuevas (2007), Eddy advective and diffusive transports of heat and salt in the Southern Ocean, J. Phys. Oceanogr., 37, 1376-1393.

MacCready, P. E., and P. B. Rhines (2001), Meridional transport across a zonal channel: Topographic localization, J. Phys. Oceanogr., 31, 1427-1439.

Maltrud, M. E., and J. L. McClean (2005), An eddy-resolving global 1/10 degrees ocean simulations, Ocean Model. 8, 31-54.

Maltrud, M. E., R. D. Smith, A. J. Semtner, and R. C. Malone (1998), Global eddy-resolving ocean simulations driven by $1985-1994$ atmospheric winds, J. Geophys. Res., 103, 30,825-30,853.

Marshall, J. C. (1981), On the parameterization of geostrophic eddies in the ocean, J. Phys. Oceanogr., 11, 257-271.

Marshall, J. C., and T. Radko (2003), Residual-mean solutions for the Antarctic Circumpolar Current and its associated overturning circulation, J. Phys. Oceanogr., 33, 2341-2354.

Marshall, J., D. Olbers, H. Ross, and D. Wolf-Gladrow (1993), Potential vorticity constraints on the dynamics and hydrography of the Southern Ocean, J. Phys. Oceanogr., 23, 465-487.

McWilliams, J. C., and J. S. Chow (1981), Equilibrium geostrophic turbulence I: A reference solution in a beta plane channel, $J$. Phys. Oceanogr., 11, 921-949.

McWilliams, J. C., W. R. Holland, and J. S. Chow (1978), A description of numerical Antarctic Circumpolar Currents, Dyn. Atmos. Ocean, 2, 213-291.

Munk, W. H., and E. Palmén (1951), Note on the dynamics of the Antarctic Circumpolar Current, Tellus, 3, 53-55.

Olbers, D. (2005), On the role of eddy mixing in the transport of zonal ocean currents, in Marine turbulence. Theories, Observations, and Models, edited by, H. Baumert, J. Simpson, and J. Sündermann, 630 pp, Cambridge University Press, Cambridge.

Olbers, D., and V. O. Ivchenko (2001), On the meridional circulation and balance of momentum in the Southern Ocean of POP, Ocean Dyn., 52, 79-93.

Olbers, D., and M. Visbeck (2005), A model of the zonally averaged stratification and overturning in the Southern Ocean, $J$ Phys. Oceanogr., 35, 1190-1205.
Panetta, R. L. (1993), Zonal jets in wide baroclinically unstable regions: Persistence and scale selection, J. Atmos. Sci., 50, 2073-2106.

Pedlosky, J. (1979), Geophysical Fluid Dynamics, Springer, New York, Inc., 624 pp.

Radko, T., and J. Marshall (2006), The Antarctic Circumpolar Current in three dimensions, J. Phys. Oceanogr., 36, 651-669.

Reznik, G. M., and V. Zeitlin (2006), Resonant excitation of Rossby waves in the equatorial waveguide and their nonlinear evolution, Phys. Rev. Lett., 96, 034502, doi:10.1103/PhysRevLett.

Rhines, P. B. (1975), Waves and turbulence on a beta-plane, $J$. Fluid Mech., 69, 417-443.

Rhines, P. B. (1994), Jets, Chaos, 4, 313-339.

Richards, K. J., N. A. Maximenko, F. O. Bryan, and H. Sasaki (2006), Zonal jets in the Pacific Ocean, Geophys. Res. Lett., 33, LO3605, doi:10.1029/2005GL024645.

Richardson, G., M. R. Wadley, K. J. Heywood, D. P. Stevens, and H. T. Banks (2005), Short-term climate response to a freshwater pulse in the Southern Ocean, Geophys. Res. Lett., 32, L03702.1L03702, doi:10.1029/2004GL021586.

Rintoul, S. R., C. Hughes, and D. Olbers (2001), The Antarctic Circumpolar Current System, in Ocean Circulation and Climate, edited by G. Siedler, J. Church, and J. Gould, pp. 271-302, Academic, New York.

Schouten, M. W., and R. P. Matano (2006), Formation and pathways of intermediate water in the Parallel Ocean Circulation Model's Southern Ocean, J. Geophys. Res., 111, C06015, doi:10.1029/2004JC002357.

Semtner, A. J., Jr. and R. M. Chervin (1988), A simulation of the Global Ocean circulation with resolved eddies, J. Geophys. Res., 93, 15,502-15,522.

Semtner, A. J., Jr. and R. M. Chervin (1992), Ocean general circulation from a global eddy-resolving model, J. Geophys. Res., 97(C4), 5493-5550.

Sinha, B., and K. J. Richards (1999), Jet structure and scaling in Southern Ocean Models, J. Phys. Oceanogr., 29, 1143-1155.

Smith, K. S., G. Boccaletti, C. C. Henning, I. Marinov, C. Y. Tam, I. M. Held, and G. K. Vallis (2002), Turbulent diffusion in the geostrophic inverse cascade, J. Fluid Mech., 469, 13-48.

Smith, R. D., J. K. Dukowicz, and R. C. Malone (1992), Parallel ocean general circulation modeling, Physica D, 60, 38-61.

Sokolov, S., and S. R. Rintoul (2002), Structure of Southern Ocean at $140^{\circ}$ E., J. Mar. Syst., 37, 151-184.

Sokolov, S., and S. R. Rintoul (2007), Multiple jets of the Antarctic Circumpolar Current South of Australia, J. Phys. Oceanogr., 37, 1394-1412.

Stammer, D. (1998), On the eddy mixing and mean flow properties, J. Phys. Oceanogr., 28, 727-739.

Stevens, D. P., and V. O. Ivchenko (1997), The zonal momentum balance in an eddy-resolving general-circulation model of the Southern Ocean, Q. J. Royal Meteor. Soc., 123, 929-951.

The FRAM group (1991), Initial results from a fine resolution model of the Southern Ocean, Eos Trans. AGU, 72, 174-175.

Treguier, A. M., and J. C. McWilliams (1990), Topographic influences on wind-driven, stratified flow in a $\beta$-plane channel: An idealized model for the Antarctic Circumpolar Current, J. Phys. Oceanogr., 20, 321-343. 
Treguier, A. M., and R. L. Panetta (1994), Multiple zonal jets in a quasigeostrophic model of the Antarctic Circumpolar Current, $J$. Phys. Oceanogr., 24, 2263-2277.

Vallis, G. K. (2006), Atmospheric and Oceanic Fluid Dynamics. Fundamentals and Large-Scale Circulation, Cambridge University Press, Cambridge, 770 pp.

Vallis, G. K., and M. E. Maltrud (1993), Generation of mean flows and jets on a beta plane and over topography, J. Phys. Oceanogr., 23, 1346-1362.

Webb, D., B. de Cuevas, and A. Coward (1998), The first main run of the OCCAM global model, Southampton Oceanography Centre, Internal Report, No 34. SOC, UK.

Wells, N. C., V. O. Ivchenko, and S. E. Best (2000), Instabilities in the Agulhas Retroflection Current system: A comparative model study, J. Geophys. Res., 105, 3233-3241.
Wolff, J.-O., and D. J. Olbers (1989), The dynamical balance of the Antarctic Circumpolar Current studied with an eddy-resolving quasi-geostrophic model, in Mesoscale-Synoptic Coherent Structures in Geophysical Turbulence, edited by J. C. J. Nihoul and B. M. Jamart, pp. 435-458, Elsevier, Amsterdam.

Wolff, J.-O., E. Maier-Reimer, and D. J. Olbers (1991), Winddriven flow over topography in a zonal $\beta$-plane channel: A quasigeostrophic model of the Antarctic Circumpolar Current, $J$. Phys. Oceanogr., 21, 236-264.

Yuan, X., and D. G. Martinson (2000), Antarctic sea ice extent variability and its global connectivity, J. Clim., 13, 1697-1717.

V.O. Ivchenko, National Oceanography Center, Southamptom, UK. (voi@noc.soton.ac.uk) 